\title{
Large-Scale Gas Flows and Streaming Motions in Simulated Spiral Galaxies
}

\author{
F. G. Ramón-Fox ${ }^{1,2 \star}$ and I. A. Bonnell ${ }^{1}$ \\ ${ }^{1}$ Scottish Universities Physics Alliance (SUPA), School of Physics and Astronomy, University of St. Andrews, North Haugh, St. Andrews, Fife KY16 9SS, UK \\ ${ }^{2}$ AIM, CEA, CNRS, Université Paris-Saclay, Université Paris Diderot, Sorbonne Paris Cité, 91191 Gif-sur-Yvette, France
}

Accepted XXX. Received YYY; in original form ZZZ

\begin{abstract}
From a galactic perspective, star formation occurs on the smallest scales within molecular clouds, but it is likely initiated from the large scale flows driven by galactic dynamics. To understand the conditions for star formation, it is important to first discern the/mechanisms that drive gas from large-scales into dense structures on the smallest scales of a galaxy. We present high-resolution smoothed particle hydrodynamics simulations of two model spiral galaxies: one with a live stellar disc ( $N$-body) and one with a spiral potential. We investigate the large-scale flows and streaming motions driven by the simulated spiral structure. We find that the strength of the motions in the radial direction tends to be higher than in the azimuthal component. In the $\mathrm{N}$-body model, the amplitude of these motions decreases with galactocentric radius whereas for the spiral potential, it decreases to a minimum at the corotation radius, and increases again after this point. The results show that in both simulations, the arms induce local shocks, an increase in kinetic energy that can drive turbulence and a means of compressing and expanding the gas. These are all crucial elements in forming molecular clouds and driving the necessary conditions for star formation.
\end{abstract}

Key words: methods: numerical - ISM: kinematics and dynamics - galaxies: ISM - galaxies: kinematics and dynamics - galaxies: spiral

\section{INTRODUCTION}

Molecular cloud formation and the triggering of star formation in galaxies results from the interaction of different physical processes operating from galactic scales to cloud scales, ranging from spiral arms and large-scale gravitational instabilities to cloudto-cloud interactions, agglomeration, and collapse within clouds (e.g. Elmegreen 2002; Bournaud et al. 2010; Burkert 2017). To understand molecular cloud formation from a galactic perspective, it is necessary to study the mechanisms that drive neutral gas from the large scales into dense structures on small scales taking into account the entire galactic context

Spiral galaxies show a wide variety of morphologies ranging from well-defined grand desígn arms to flocculent structures. Galactic-scale gas flows are sensitive to the physical nature of the underlying spiral arms. Mainly, two models have been proposed to describe these structures: spiral density waves (SDW; e.g. Lin \& Shu 1964; Bertin \& Lin 1996; Shu 2016) and dynamic or material arms (e.g. Goldreich \& Lynden-Bell 1965; Julian \& Toomre 1966; Sellwood \& Carlberg 1984). In the first one, the arm is viewed as a density wave travelling in the stellar disc that rotates with a relatively constant pattern speed. It extends over the entire galaxy

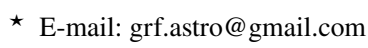

and its rotation speed is independent of the individual stellar and gas motions. It is expected to dissipate on long timescales of $\sim 1$ Gyr. In the second, the arms are described as dynamic features with variable amplitudes that grow, evolve, and eventually dissipate on shorter timescales. The number of arms and pattern speed vary with radius. See Dobbs \& Baba (2014) for a review. The differences in the gas flows predicted by each model can translate into variations in the formation and evolution of molecular clouds in an arm.

In the SDW scenario, gas flows into an arm, it forms a shock (e.g. Fujimoto 1968; Roberts 1969; Shu et al. 1972), and it eventually leaves the arm due to its relative motion with respect to the arm (except at the corotation radius). Several works using this model show that, as gas flows into the arm, its velocity normal to the arm increases, then falls sharply at the shock's location, and increases again as gas leaves the arm. The tangential component decreases and then increases after passing through the shock region. This model predicts that molecular clouds and star forming regions should be observed after the shock (e.g. Roberts 1969; Gittins \& Clarke 2004; Sormani et al. 2017).

In the dynamic arm scenario, the flows can be more complex. The $N$-body simulations of Wada et al. (2011); Baba (2015); Baba et al. (2016) show that gas flows from both sides of an arm, forming a high density region in its centre. Wada et al. (2011) and Baba et al. (2016) find streaming motions with noticeable arm-to-arm 
variations, but still showing some periodicity. However, these simulations differ with respect to spiral potential simulations on the location of the shock and the turning point of the streaming motions with respect to an arm's position. They also show that an arm rotates with a speed close to the galaxy's rotation curve instead of having a constant pattern speed. This means that once the gas settles in an arm, it will likely stay there until the arm dissipates or it escapes with the help of energy injected by feedback. In this model, molecular clouds are expected to be centred in an arm (e.g. Wada et al. 2011; Baba et al. 2016).

Both models predict significant non-circular or streaming motions for the gas in an arm, but with different velocity profiles, which provides an observational diagnostic of each scenario (Baba et al. 2016). Molecular clouds and star forming regions can be used to probe these motions in the Milky Way. Many works have observed high-mass star forming regions to study molecular cloud kinematics in Galactic spiral arms: for example, Choi et al. (2014) and Sato et al. (2014) find that, in the Perseus and Scutum arms, respectively, the clouds are on average moving towards the Galactic centre and slightly slower than the circular velocity; Hachisuka et al. (2015) find a similar trend in the galactocentric component in the Outer Arm. These observations are consistent with a Milky Way spiral arm simulation by Ramón-Fox \& Bonnell (2018). A recent Galactic kinematic analysis by Tchernyshyov et al. (2018), combining $\mathrm{H}_{\mathrm{I}}, \mathrm{CO}$, and dust data, suggests that the Perseus Arm has the kinematic features of a dissipating arm as predicted by $\mathrm{N}$ body simulations, differing with the SDW interpretation proposed by Choi et al. (2014), which shows the importance of characterising the kinematic signatures of spiral arms.

From an extragalactic perspective, Schmidt et al. (2016) found significant galactocentric motions ranging from a few to several $\sim 10 \mathrm{~km} \mathrm{~s}^{-1}$ in the $\mathrm{H}_{\mathrm{I}}$ kinematics of a galaxy sample from the THINGS survey (Walter et al. 2008). Using CO data from M51, Meidt et al. (2013) found streaming motions in the range of $\approx \pm 30$ $\mathrm{km} \mathrm{s}^{-1}$ and Colombo et al. (2014) measured values as high as $\approx 70$ $\mathrm{km} \mathrm{s}^{-1}$ for the $m=2$ spiral and lower than $30 \mathrm{~km} \mathrm{~s}^{-1}$ for higher modes. Some observations of molecular clouds in M51 suggest that streaming motions affect their star formation efficiency (Meidt et al. 2013).

A better understanding of the dependence of large-scale flows on galactic structure can improve our view of the gas dynamics leading to molecular cloud formation and the conditions for star formation from a galactic perspective (e.g. Bonnell et al. 2013; Meidt et al. 2018). It is also important to develop simulations that help to bridge the gap between those modelling large-scale processes (e.g. Bournaud et al. 2010; Bonnell et al. 2013) and those resolving star formation on smaller scales (e.g. Smilgys \& Bonnell 2017).

In this paper, we characterise the differences in the large-scale flows, streaming motions, and velocity dispersion between different spiral arm models. We perform $\mathrm{N}$-body and spiral potential simulations with rotation curves matching as closely as possible; we set the spiral potential to represent as close as possible the amplitude of the arms in the $\mathrm{N}$-body model for a more direct comparison of both models. We perform simulations with higher resolution in comparison to previous numerical work. This paper is divided as follows: $\$ 2$, introduces the numerical code and the methods applied to initialise the galaxy models; $\$ 3$ presents the results comparing both models; $\S 4$ presents a discussion and $\S 5$ summarises our results.

\section{METHODS AND SIMULATIONS}

\subsection{Numerical Code}

We use the smoothed particle hydrodynamics (SPH) method (Gingold \& Monaghan 1977; Monaghan 1992) to simulate the gas dynamics. We use the code sphNG, which implements the formulation of Benz et al. (1990); Bate et al. (1995). SPH represents a gas with a particle distribution, where each one has a fixed mass and a smoothing length $h$. This parameter sets the spatial resolution and is calculated by making sure that each particle has approximately 50 neighbours. In order to treat shock regions, the code uses an artificial viscosity with the parameters $\alpha=1$ and $\beta=2$ (Monaghan \& Lattanzio 1985; Monaghan 1992). The gravitational forces are calculated with a tree code for the long-range component and direct neighbour forces for the short-range contribution. The thermal physics implements the cooling function of Koyama \& Inutsuka (2002), which includes losses due to atomic, molecular, and dust emission. Shock heating is also included. The internal energy is integrated implicitly following the method of Vázquez-Semadeni et al. (2007). For further details refer to Bonnell et al. (2013) and Lucas et al. (2013).

\section{$2.2 N$-body galaxy model}

\subsubsection{Galaxy Components and Density Profiles}

We first perform simulations of an $N$-body model composed of a stellar disc, a central bulge, and a gas disc. A dark matter halo is included as a static potential, which allows to direct the computational efforts to the disc dynamics. The disc has an exponential-isothermal density profile (e. g. Freeman 1970; van der Kruit \& Searle 1981; van der Kruit \& Freeman 2011) given by:

$\rho_{d}(R, z)=\frac{M_{d}}{4 \pi R_{d}^{2} z_{d}} \exp \left(-\frac{R}{R_{d}}\right) \operatorname{sech}^{2}\left(\frac{z}{z_{d}}\right)$,

where $R_{d}$ and $z_{d}$ are the radial and vertical scale lengths, respectively. We assume that the disc's total mass is given by $M_{d}=M_{\mathrm{gas}}+M_{\star}$, where $M_{\mathrm{gas}}$ and $M_{\star}$ are the total gas and stellar masses, respectively. The gas and stellar mass fractions are expressed as $f_{\text {gas }}=M_{\text {gas }} / M_{d}$ and $f_{\star}=M_{\star} / M_{d}$, respectively. This approach assumes that both the gas and stellar discs have the same $R_{d}$. We adopt it for its simplicity for generating the initial conditions. The bulge is modelled by the Hernquist (1990) profile:

$\rho_{b}(r)=\frac{M_{b}}{2 \pi r_{b}^{3}} \frac{1}{\left(r / r_{b}\right)\left(1+r / r_{b}\right)^{3}}$,

Where $M_{b}$ and $r_{b}$ are the bulge's mass and scale radius, respectively. The static dark matter halo follows a Navarro-Frenk-White (NFW, Navarro et al. 1997) profile given by:

$\rho_{h}(r)=\frac{\rho_{0}}{\left(r / r_{h}\right)\left(1+r / r_{h}\right)^{2}}$,

where $r_{h}$ is the scale radius, $\rho_{0}$ is the central density.

The model galaxy's physical parameters are summarized in Table 1 and Figure 1 (black curve) shows the model rotation curve. The Toomre parameter at $R=1.5 R_{d}$ is set to $Q=1.5$ and the orbital period at $R=2 R_{d}$ is $T_{\text {orb }}=284 \mathrm{Myr}$. The parameters are motivated by previous observations and models of M33's rotation curve (e.g. Regan \& Vogel 1994; Corbelli \& Salucci 2000; Seigar 2011; Hague \& Wilkinson 2015; Kam et al. 2015). M33 is a fairly low-mass spiral galaxy close to the Milky Way. Its inclination provides an advantageous point of view to map the ISM and the location 
Table 1. $N$-body Model Galaxy Parameters

\begin{tabular}{lc}
\hline Disc \\
\hline$M_{d}\left[10^{9} M_{\odot}\right]$ & 9.0 \\
$R_{d}[\mathrm{kpc}]$ & 2.5 \\
$z_{d}[\mathrm{kpc}]$ & 0.2 \\
$f_{\star}$ & $85 \%$ \\
$f_{\text {gas }}$ & $15 \%$ \\
\hline Bulge & \\
\hline$M_{b}\left[10^{8} M_{\odot}\right]$ & 3.0 \\
$r_{b}[\mathrm{kpc}]$ & 0.4 \\
\hline Halo & \\
\hline$M_{h}\left[10^{11} M_{\odot}\right]$ & 5.7 \\
$R_{h}[\mathrm{kpc}]$ & 33.8 \\
$c$ & 4.0 \\
\hline
\end{tabular}

of molecular clouds with respect to the galaxy's stellar structure. Recent surveys have provided detailed observations of M33's ISM components (e.g. Druard et al. 2014; Kam et al. 2015; Koch et al. 2018, 2019), which motivates a more detailed comparison with simulations. We do not aim to exactly reproduce M33 in our simulations. Our model may be viewed as a representation of low-mass galaxies.

The initial conditions are generated with the method of McMillan \& Dehnen (2007), which is implemented in the public code MKGALAXY $^{1}$. It produces a self-consistent galaxy model composed of $N$-body realizations of a halo, a disc, and a bulge. This method has the advantage of producing a stellar disc with velocities sampled from a distribution function representative of the kinematics of a disc, rather than assuming a local Gaussian distribution (Dehnen 1999). The resulting disc has an azimuthal velocity distribution following the galaxy's rotation curve.

Since the mass integral of the NFW profile does not converge, MKGALAXY includes a truncation function $T(r)$ at large radii in equation (3) to allow for a finite mass. $T(r)$ is defined as:

$T\left(r / r_{t}\right)=\frac{2}{\operatorname{sech}\left(r / r_{t}\right)+1 / \operatorname{sech}\left(r / r_{t}\right)}$.

We set $r_{t}=62 \mathrm{kpc}$ in our model. This parameter is relevant for simulations using the live halo produced by the code. We include this parameter for completeness, but as previously described, we use a static halo for our simulations.

To finalize our setup, we extract only the disc and bulge particles from the initial conditions generated by MKGALAXY and evolve them in a static non-truncated NFW halo. We specified parameters for MKGALAXY that produce a halo potential in the disc region that approximates that of the non-truncated NFW profile. We tested this setup by initializing a model with 500000 disc and 10000 bulge and evolving it in a static halo for approximately $4 T_{\text {orb }}$. This showed that the average surface density profile was preserved during this period. Although M33's røtation curve is bar-unstable (Dobbs et al. 2018; Sellwood et al.2019), this test did not form one during this period, likely due to the static halo. The above method does not generate initial conditions for the gas. The gas intialization is described in the following sub-section.

1 MKGALAXY can be downloaded from the NEMO Stellar Dynamics Toolbox (Teuben 1995): https://bima.astro.umd.edu/nemo/

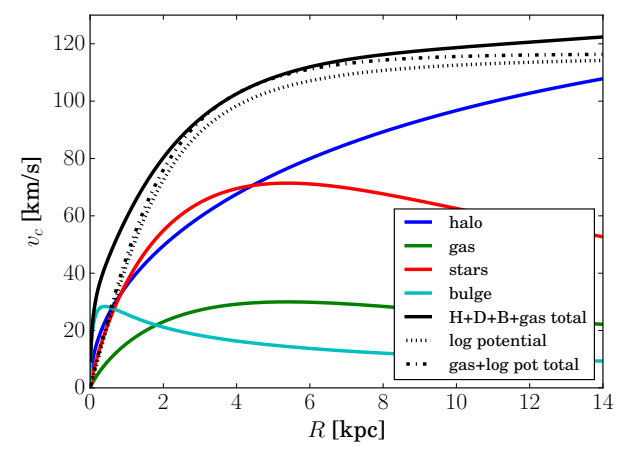

Figure 1. Total rotation curve for the $N$-body model (black), which results from the contribution of the halo (blue), stellar disc (red), gas disc (green), and bulge (cyan); rotation curve for the logarithmic potential (dotted line) and total rotation curve used for the spiral potential simulation (dotted-dashed line).

\subsubsection{Adding a Gas Component}

The full galaxy model is built by first generating a disc containing the total number of particles $\left(N_{\text {disc }}=N_{\star}+N_{\text {gas }}\right.$, where $N_{\star}$ and $N_{\text {gas }}$ are the number of stellar and gas particles, respectively) and the total combined stellar and gas mass $\left(M_{d}=M_{\star}+M_{\text {gas }}\right)$. This disc is then divided into gas and stellar components. We specify the number of gas and stellar particles such that $N_{\text {disc }}$ is preserved and the particle másses are assigned as follows: $m_{\text {gas }}=M_{\text {gas }} / N_{\text {gas }}$ and $m_{\star}=M_{\star} / N_{\star}$, for the gaseous and stellar components, respectively. After this step, the particle distribution has two separate components, but the total mass and particle numbers are preserved. The positions and velocities are those from the initial condition generator of $\$ 2.2 .1$, which do not satisfy the vertical hydrostatic balance condition for the gas. This means that the system has to evolve for some time to allow the gas to stabilize in the galaxy's potential (e.g Dobbs et al. 2010).

To initialise the disc in hydrostatic balance, we find the vertical equilibrium density profile for the gas by solving the hydrostatic balance equation:

$\frac{1}{\rho_{g}} \frac{\partial P}{\partial z}+\frac{\partial \Phi}{\partial z}=0$

coupled to the Poisson equation:

$\nabla^{2} \Phi=4 \pi G\left(\rho_{h}+\rho_{\star}+\rho_{g}\right)$,

where $\rho_{h}$ is the dark matter halo density, $\rho_{\star}$ is the density of the stellar components, and $\rho_{g}$ is the gas density; $P$ is the gas pressure and $\Phi$ is the galaxy's gravitational potential.

As described in Wang et al. (2010), by assuming a thin disc approximation and an isothermal equation of state: $P=\rho_{g} c_{s}^{2}$, the problem is reduced to the following equations:

$\rho_{g}(R, z)=\rho_{g}(R, 0) e^{-1 / c_{s}^{2}\left[\Delta \Phi_{\mathrm{gal}}(R, z)+\Delta \Phi_{g}(R, z)\right]}$,

$\frac{d^{2} \Phi_{g}}{d^{2} z}=4 \pi G \rho_{g}$

where $c_{s}$ is the sound speed; $c_{s}=\left(k_{B} T /\left(\mu m_{p}\right)\right)^{1 / 2}$, where $k_{B}$ is the Boltzmann constant, $T$ is the gas temperature, $\mu$ is the mean molecular weight and $m_{p}$ is a proton's mass. For a shorter notation, we define $\Delta \Phi(R, z)=\Phi(R, z)-\Phi(R, 0)$, which is the potential 
difference between a vertical position $z$ and the mid-plane. In this notation, $\Delta \Phi_{\text {gal }}$ denotes the contribution of the halo and stellar components and $\Delta \Phi_{g}$, that of the gas. This approach assumes pure thermal support and it does not use an effective sound speed to account for turbulent motions.

To obtain the density profile, equation (7) is first substituted in equation (8) to have a second order non-linear differential equation for the gas potential $\Phi_{g}$. Once this is known, the density profile is calculated with equation (7). The boundary conditions are $\Phi(R, 0)$, $d \Phi /\left.d z\right|_{z=0}$, and $\rho_{g}(R, 0)$. The gas density is coupled to the surface density profile by:

$\Sigma(R)=\int_{-\infty}^{\infty} \rho_{g}(R, z) d z$,

This is evaluated with numerical integration. Since the correct midplane density profile is not known, it has to be solved iteratively by coupling equations (7), (8), and (9) (see Wang et al. 2010 for a full description).

For the non-gaseous term in equation (7), the potentials for the halo, disc, and bulge are needed. For the halo and bulge models, algebraic expressions are available (Binney \& Tremaine 2008). For the exponential disc, it is possible to express the potential at a given point as (Cuddeford 1993; Binney \& Tremaine 2008):

$\Phi(R, z)=-\frac{4 G \Sigma_{0}}{R_{d}} \int_{-\infty}^{\infty} \zeta\left(z^{\prime}\right) d z^{\prime} \int_{0}^{\infty} \sin ^{-1}(G(a)) a K_{0}\left(a / R_{d}\right) d a$

where,

$G(a)=\frac{2 a}{\sqrt{z^{2}+(a+R)^{2}}+\sqrt{z^{2}+(a-R)^{2}}}$,

$\zeta(z)$ is a normalised function corresponding to the vertical part of the profile $\left(\propto \operatorname{sech}^{2}\left(z / z_{d}\right)\right), K_{0}$ is a modified Bessel function, and $\Sigma_{0}$ is the central surface density.

The numerical solution for $\rho_{g}(R, z)$ (see equations (7) and (8)) is obtained just for a discrete set of galactocentric radii. At each radius, the vertical potential for the disc is calculated using equation (10) for a set of vertical positions to build an interpolating function that can be used for a faster calculation at all values of $z$. Then, the total potential used in equation (7) includes this interpolating function plus the halo and bulge terms to specify the galaxy's potential.

The resulting vertical density profile is very similar to a sech ${ }^{2}$ profile, but with a scale height dependent on radius $\left(h_{g}=h_{g}(R)\right)$. In order to initialise the gas particles' positions, the vertical density profile would need to be calculated at the radial position of each particle in order to sample its position from this profile. We can simplify our approach by taking advantage of the fact that the initial conditions generator already samples the particles' vertical position from a sech ${ }^{2}$ with a constant scale height. For the particles that we select to be gas, we can rescale their vertical position in terms of assuming a sech ${ }^{2}$ profile with the local scale height value. To have a continuous function of scale height as a function of radius, we first solve the hydrostatic balance equation at a fixed set of radii and use the results to build a interpolating function $h_{g}(R)$. We use this function to initialise the vertical position of the gas particles. Finally, the gas velocities are sampled from the circular velocity function.

We set the gas and stellar disc particle numbers to $N_{\text {gas }}=$ $30 \times 10^{6}$ and $N_{\star}=2 \times 10^{6}$, respectively. The bulge has $N_{b}=78420$ particles. In terms of individual particle masses, $m_{g}=45 \mathrm{M}_{\odot}$ for the gas particles and $m_{\star}=m_{b}=3825 \mathrm{M}_{\odot}$, for the stellar and bulge

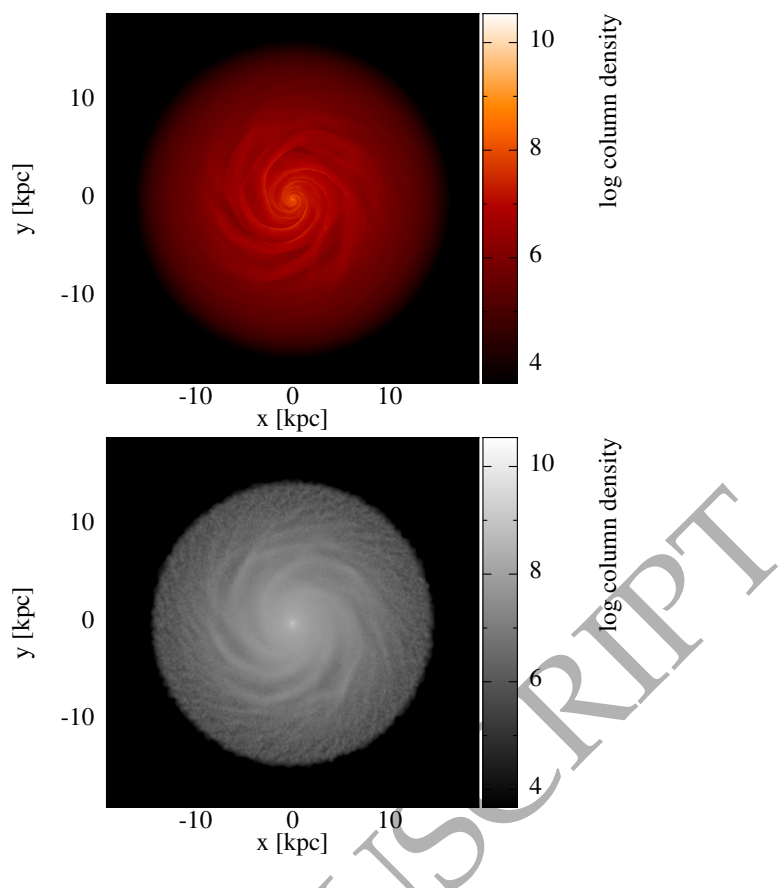

Figure 2. Surface density map of the gas (top panel) and stellar (bottom panel) components for the $N$-body model at the time chosen to start the full simulation with heating and cooling. The column density is in $\left[\mathrm{M}_{\odot} / \mathrm{kpc}^{2}\right]$.

particles. A softening length of $5 \mathrm{pc}$ is used for the gravitational force for all the particles in the simulation. The gas is initialized with $T=10^{4} \mathrm{~K}$. The model is evolved isothermally for $t \approx 747$ $\operatorname{Myr}(\approx 2.6$ orbital periods) to allow the spiral structure to form (Figure 2 shows the gas and stellar components at this time). Then, the heating and cooling mechanisms are switched on and the system is allowed to evolve for an additional period of $\approx 42 \mathrm{Myr}$, but our analysis is performed at $20 \mathrm{Myr}$.

\subsection{Spiral arm potential galaxy model}

We also perform simulations of a galaxy model based on the analytic expressions for the gravitational potential of the stellar and dark matter components in order to compare it with the $N$-body model of $\$ 2.2$. The galactic potential is represented by a combination of an axisymmetric term plus a term for the spiral arms: $\Phi_{\text {gal }}=$ $\Phi_{\text {axi }}+\Phi_{\text {spiral }}$. The first term is given by a logarithmic potential (e.g. Binney \& Tremaine 2008):

$\Phi_{\text {axi }}(R, z)=\frac{1}{2} v_{0}^{2} \log \left(R_{0}^{2}+R^{2}+\left(z / z_{q}\right)^{2}\right)$

where $v_{0}$ is the velocity parameter, $R_{0}$ is the characteristic radius, and $z_{q}$ is the vertical scale factor, and $R, z$ are the radial (galactocentric) and vertical coordinates. This produces a flat rotation curve for $R \gg R_{0}$. The gas disc in this model has the same contribution to the rotation curve as the one in the $N$-body model.

We use the potential of Cox \& Gómez (2002) for the spiral arms, which is expressed as:

$\Phi_{\text {spiral }}(R, \theta, z, t)=\sum_{n} A_{n}(R, z) \cos (n \Gamma(R, \theta, t))$,

where $A_{n}$ is the perturbation's amplitude and $\Gamma$ determines the arm's geometry, which is given by:

$\Gamma(R, \theta, t)=N\left(\theta+\Omega_{p} t-\frac{\ln \left(R / R_{\mathrm{ref}}\right)}{\tan \alpha}-\theta_{p}\right)$ 

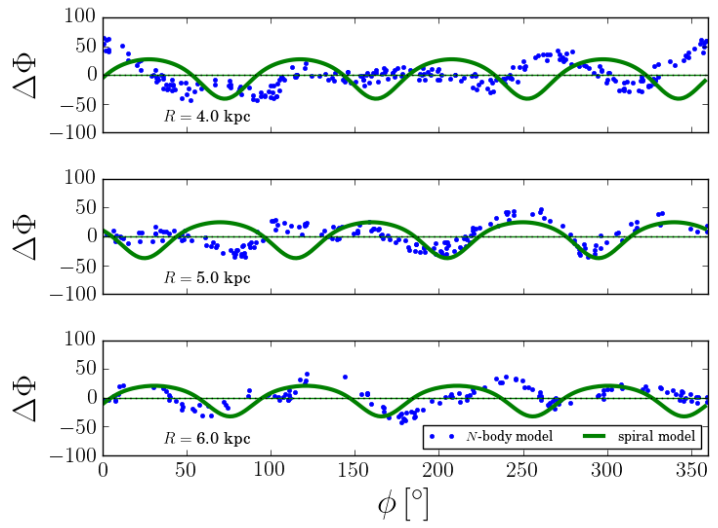

Figure 3. Relative potential $\Delta \Phi$ (in code units) as a function of azimuth (in radians) for the $N$-body arms (blue dots) and spiral potential (green curve) arms. $\Delta \Phi$ is defined as: $\Delta \Phi=\Phi(\phi)-\Phi_{\text {mean }}$, where $\Phi_{\text {mean }}$ is the mean potential at the specified radius. The values shown for the $N$-body model correspond to a selection of stellar particles inside an annulus $100 \mathrm{pc}$ wide centred on each radius and within $|z|<2$ pc.

In equation (13), $\Phi_{\text {spiral }}$ is the summation of $n$ cosine terms. We use the standard formulation of Cox \& Gómez (2002) which uses 3 terms ( $n=3)$. The form of $A_{n}(R, z)$ is given in equation (8) of Cox \& Gómez (2002); this potential produces a density distribution that falls exponentially in the form $\exp \left(-\left(R-R_{\text {ref }}\right) / R_{S}\right)$, where $R_{S}$ is a scale radius and $R_{\text {ref }}$ is a reference radius (appearing in equation 14) that allows us to specify the profile using the overall density at position different than $R=0$; also, in equation (14), $\theta_{p}$ controls the initial phase of the pattern at $R_{\text {ref }}$.

In our model, the number of arms is $N=4$, the pitch angle is $\alpha=15^{\circ}$, and the pattern speed is $\Omega_{p}=23 \mathrm{~km} \mathrm{~s}^{-1} \mathrm{kpc}^{-1} . \Omega_{p}$ is chosen to approximately match that of the $N$-body model at $R \approx 4 \mathrm{kpc}$. Although the number of arms is fixed, these parameters ensure that the arm potential amplitude approximately matches that of the $N$-body model in the range $4<R<6 \mathrm{kpc}$ (see Figure 3 ). The gaseous component is assumed to have an exponential surface density profile: $\Sigma(R)=\Sigma_{0} e^{-R / R_{d}}$, where $\Sigma_{0}$ is the central surface density and $R_{d}$ is the scale radius; the central surface density is given by $\Sigma_{0}=M_{g} /\left(2 \pi R_{d}^{2}\right)$.

We assign $M_{g}=f_{\text {gas }} M_{d}$ and $R_{d}$ the same values of Table 1 to have the same gas disc parameters as the $N$-body simulation. This gas disc is initialized with $30 \times 10^{6}$ particles and $T=10^{4} \mathrm{~K}$. It is evolved isothermally and without self-gravity for $\approx 471 \mathrm{Myr}$ before including heating and cooling. The disc is subject to a logarithmic potential with the following parameters: $v_{0}=220 \mathrm{~km} \mathrm{~s}^{-1}, R_{0}=2.5$ $\mathrm{kpc}$, and $z_{q}=0.7$. For this step, the initial vertical gas distribution is calculated using only equation (7) from $\$ 2.2$. The mid-plane density is obtained from equation (9) given the surface density profile. We take this approach for its reduced computational cost for initializing the disc. At $t \approx 471 \mathrm{Myr}$, self-gravity, heating and cooling are activated. Once the gas self-gravity is included, it adds a contribution to the rotation curve. Thus to preserve its overall shape of the rotation curve, the parameter $v_{0}$ is slightly reduced to $116 \mathrm{~km} \mathrm{~s}^{-1}$. The total rotation curve with self-gravity included is shown in Figure 1. This choice of parameters gives a corotation radius at $R_{c}=4.62 \mathrm{kpc}$. A resolution test is presented in Appendix $\mathrm{A}$ and results of lower resolution versions of both the $\mathrm{N}$-body and spiral potential models can be found in Ramón-Fox (2019) and Forgan et al. (2018).

\section{RESULTS}

\subsection{Evolution of the Disc Structure and Gas Morphology}

In this section, we present face-on maps of the gas surface density of the model galaxies, which show the evolution of the disc's morphology after including heating and cooling. This allows us to examine from a galactic perspective where cloud-like structures are forming. Figure 4 shows the $N$-body (left panels) and spiral potential (right panels) simulations at $t=20.4 \mathrm{Myr}$ after including heating/cooling. The galaxies have a counter clockwise rotation. We choose this time to examine the gas dynamics in the first stages of cloud formation.

Both simulations show that high-density regions first form in the galaxy's inner regions and in the spiral arms. The $N$-body model shows that high-surface density gas is located at galactocentric distances as large as $3 \mathrm{kpc}$. The dense gas tracing the spiral arms extends to larger galactocentric distances (top left panel of Figure 4). On the other hand, the spiral potential simulation at the same time shows that the surface density increases mostly in the arms (top right panel of Figure 4). Both simulations show that high surface density gas forms within $\approx 5 \mathrm{kpc}$ of the galaxy's centre, although this is a particular result of our models.

The lower panels of Figure 4 show zoom-in views of selected spiral arms of the $N$-body (left) and potential (right) simulations. Both simulations show that dense clouds are formed in the arms and their spacing resembles a "beads on a string" pattern. However, the $\mathrm{N}$-body simulation shows a rich variety of gas structures both in the arm and interarm regions while in the spiral potential simulation, most structure develops in the arm, with some diffuse features in the downstream side. This difference may likely be an effect of the initialization of both models. The spiral potential simulation has a much smoother surface density profile at $t=0$, so it takes longer to form substructure. In agreement with previous works (e.g. Fujimoto 1968; Roberts 1969; Clarke \& Gittins 2006; Dobbs \& Bonnell 2007; Wada 2008; Wada et al. 2011; Bonnell et al. 2013; Pettitt et al. 2016), our simulations also show that spiral arms drive the formation of dense structures resembling molecular clouds.

It is worth noting that Figure 4 qualitatively shows that the clouds in an arm of the potential simulation appear to have fairly regular spacing while those in the $\mathrm{N}$-body model have a more variable separation. This is interesting, considering that at the moment the where the heating and cooling are included, the gas in the arms of both models is not fragmented. The width of the gas arms appears thin in both models, likely an effect of the lack of feedback.

Figure 5 shows two zoom-in panels of the $N$-body simulation at $t=20.4 \mathrm{Myr}$ (upper panel) and at $t=30.5 \mathrm{Myr}$ (lower panel). The snapshots show a rich variety of structures in the dense gas in the central region and along the spiral arms. The second snapshot shows that the structures become denser due self-gravity and the lack of feedback in our simulation. It is worth noting that there are features resembling spurs and feathers in the interarm regions. See, for example, the region in the vicinity of $(X, Y)=(0,3) \mathrm{kpc}$ and $(X, Y)=(-2,3) \mathrm{kpc}$. The absence of these features in the spiral potential simulation at the time shown is likely an effect of our warm initial conditions. For example, the simulations of Dobbs \& Bonnell (2006) show that spurs tend to form when cold, dense gas passes through an arm. Ramón-Fox (2019) performed a simulation of the spiral potential model without self-gravity that show that the spurs are already formed in the galaxy's inner region at $t \approx 120$ $\mathrm{Myr}$, indicating that these features should appear later in our setup. 

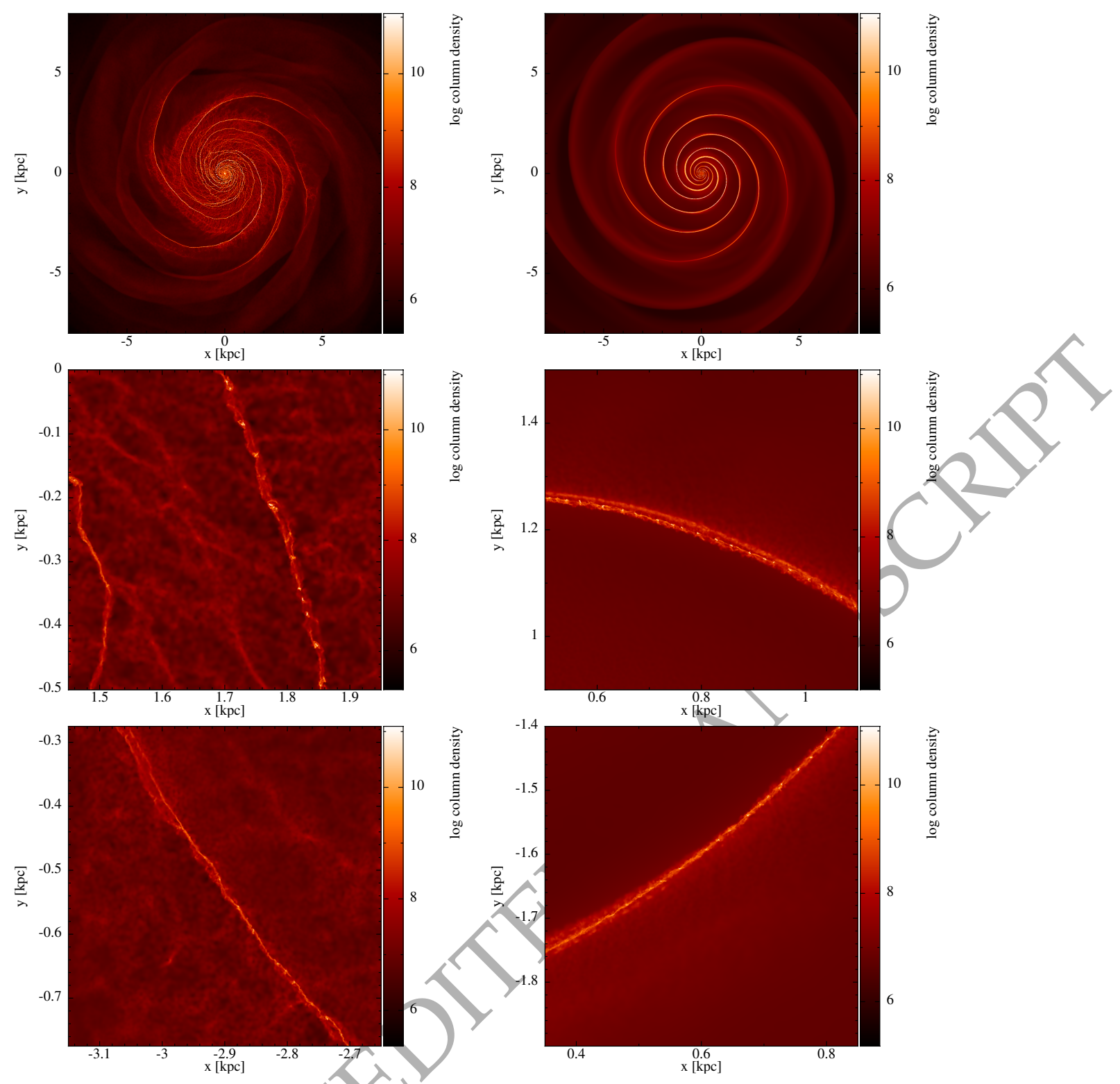

Figure 4. Surface density maps of the model galaxies at $t=20.4 \mathrm{Myr}$. The top left and right panels show global views of the $N$-body and spiral potential simulations, respectively. The middle and bottom panels show zoom-ins of two different regions of a spiral arm in the $N$-body and spiral potential simulations on the left and right, respectively. The surface density is in $\left[\mathrm{M}_{\odot} / \mathrm{kpc}^{2}\right]$. Both simulations show that clouds formed in the arms follow a string-like pattern.

\subsection{Radial Distribution of the Gas Phases}

The thermal physics of our models allows the formation of a multiphase medium. This section describes how these phases are distributed in terms of galactocentric radius and quantifies the overall change in the mass fraction of each phase as a function of time. The gas phases are specified as follows: the cold phase is defined as gas with $\rho>10 \mathrm{M}_{\odot} \mathrm{pc}^{-3}$ and $10<T<2 \times 10^{2} \mathrm{~K}$; the intermediate phase, as gas with $10^{-6}<\rho<10 \mathrm{M}_{\odot} \mathrm{pc}^{-3}$ and $10<T<5 \times 10^{3}$ $\mathrm{K}$; the warm phase is defined as gas with $\rho<10 \mathrm{M}_{\odot} \mathrm{pc}^{-3}$ and $5 \times 10^{3}<T<1.2 \times 10^{4} \mathrm{~K}$. The gas with $T>1.2 \times 10^{4} \mathrm{~K}$ is not included in our analysis because we find that most of the particles in this range trace very low-density gas near the galaxy's edges. At theselocations, the SPH smoothing lengths may be artificially enlarged as the algorithm searches over larger radii to keep the number of neighbours approximately constant. This may lead to less accu- rate density calculations at those positions. However, the fraction of mass in this range is $<2 \%$ of the total gas mass in both models. Our analysis also focuses on the inner disc dynamics.

Figure 6 shows the radial surface density profiles of these phases at $t=20.4 \mathrm{Myr}$. The top and bottom panels show the profiles of the $N$-body and spiral potential simulations, respectively. In both, the cold gas surface density decreases with radius whereas the warm gas shows a slowly decaying profile at large galatocentric distances. The $N$-body model has a higher central density of cold gas due to the bulge. The warm gas tends to have higher surface densities in the spiral potential simulation. An interesting difference is that the intermediate phase profile tends to be flat at $1<R<4 \mathrm{kpc}$ in the $N$-body model, while it has a decreasing profile in the spiral potential simulation. It is worth noting that the cold and warm surface density profiles qualitatively agree with the profiles reported 

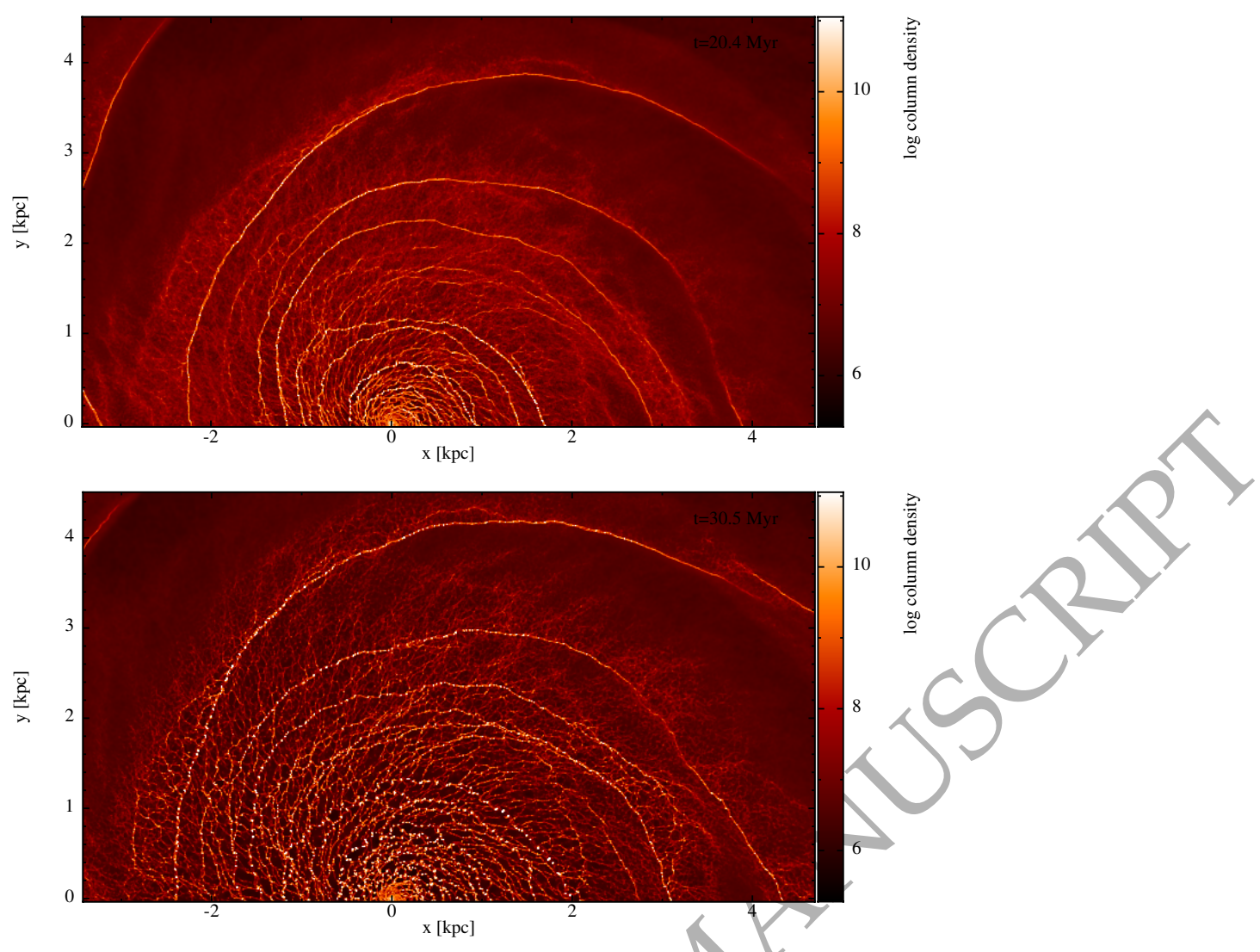

Figure 5. Zoom-in panels of the $N$-body simulation at $t=20.4 \mathrm{Myr}$ (upper panel) and $t=30.5$ Myr (lower panel), which show a rich variety morphological structure and features resembling spurs in several interarm regions. The surface density is in units of [M $\left.\odot / \mathrm{kpc}^{2}\right]$.

in Bigiel \& Blitz (2012), which show that neutral gas tends to have flat profiles while molecular gas follows exponential profiles. Gratier et al. (2010) and Druard et al. (2014) report a similar result for M33. The values of the warm gas surface density of the spiral potential simulation are close to the values reported for M33, although the values of the $N$-body model are slightly lower. The value of the central cold gas central surface density is higher than that of M33.

Figure 7 shows the mass fraction of gas in each phase for the $\mathrm{N}$ body (top panel) and the spiral potential (bottom panel) simulations. In both simulations, the cold gas fraction grows steadily with time. During this interval, the gas distribution in the N-body simulation is dominated by gas in the intermediate phase. In the spiral potential simulation, the warm phase is the most abundant one. It is worth pointing out that in this simulation, the gas from the cold phase seems to be coming from the intermediate phase. Figure 7 (bottom panel) shows that as the dense gas fraction increases (red curve) the intermediate gas phase fraction decreases (green curve). Figure 7 shows that the the $N$-body model quickly loses an important quantity of warm gas. This effect seems to be a result of the initialization of the models. Since the $N$-body model includes gas self-gravity before heating is included, this drives higher gas densities that cool faster once the heating is included.

The surface density profiles of the cold and gas phases in our simulations have qualitatively similar shapes regardless of how the axisymmetric and galactic spiral arm components are modelled, indicating a similar galactocentric distribution of the gas phases.

\subsection{Azimuthal Gas Distribution}

The gas flows across an arm establish an azimuthal density profile. Its shape and the position of its maximum density are sensitive to the nature of the flow: shocks produce sharp density jumps, with the peak density position slightly displaced from the arm's centre; converging flows produce the highest densities at the arm's centre. Figure 8 shows the gas surface density maps in cylindrical coordinates for the $N$-body (top panel) and the spiral potential (bottom panel) simulations at $t=20.4 \mathrm{Myr}$. Both simulations show a surface density enhancement in the spiral arms. The arms in the $N$-body model tend to have slight bends while the ones in the spiral potential simulation follow a smooth curve.

In the $N$-body model (top panel Figure 8 ), we first focus on the region where $R<5 \mathrm{kpc}$. When following a constant radius line (e.g. $R=4 \mathrm{kpc}$ ) in the positive $\phi$ direction, on the left side of an arm (upstream), the surface density first sharply increases and gradually decreases with azimuth on the downstream side (arm's right side in this panel). This is better visualised in the azimuthal volume density profiles in the left panel of Figure 9. The ones for $R=3 \mathrm{kpc}$ (top left) and $R=4 \mathrm{kpc}$ (second left) clearly show this. In the $N$-body model, the gas in the inner $5 \mathrm{kpc}$ rotates slightly faster than the arms, as shown in Figure 10, which plots the spiral arms' pattern speed compared to the galaxy's angular velocity. The arm pattern speed is calculated by averaging the angular velocity of the stellar surface density peaks at the selected radius. This difference in angular speed may explain the behaviour of the density profiles at $R=3$ and $4 \mathrm{kpc}$. 

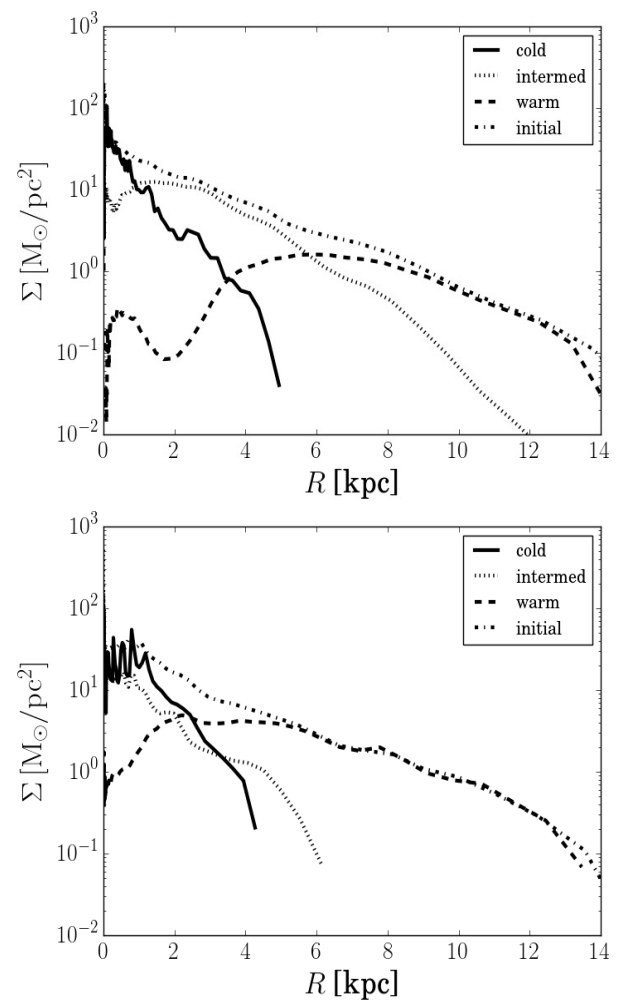

Figure 6. Gas surface density profiles of the cold (black line), intermediate (dotted line), and warm (dashed line) phases in the $N$-body (top) and spiral potential simulations (bottom) at $t=20.4 \mathrm{Myr}$. The dashed-dotted line is the total surface density at $t=0$.

A similar profile still forms at $R=5 \mathrm{kpc}$, where the local pattern speed is close to the frequency of the rotation curve.

Figure 10 also shows that at galactocentric distances between 6 and $10 \mathrm{kpc}$, the angular frequency of the rotation curve is slightly lower than the pattern speed of the arms. The arms at $R=10 \mathrm{kpc}$ almost corotate with the galaxy. On a constant radius line at $R=7$ $\mathrm{kpc}$ in the positive $\phi$ direction in Figure 8, there is a strong density contrast at the location of several arms and a high surface density tail on the arm's left side. This is different to the behaviour of the arms at $R<6 \mathrm{kpc}$ in the same map. This can be expected from gas moving slower than the arm's rotation speed. However, the small difference between the pattern speed and the gas rotation speed and the features of the volume density profile at $R=7 \mathrm{kpc}$ (bottom left panel of Figure 9) suggests that the gas is mostly clumping in the stellar arms due the low relative velocity between the arm and gas. The sharp surface density contrasts at $R>6 \mathrm{kpc}$ seem to be an effect of the projection of strong volume density changes in some arms. The four profiles of the $\mathrm{N}$-body simulation in Figure 9 show that the peak densities in the arms systematically decrease with $R$, which results from the falling amplitude of the arms' potential (Figure 15 plots the potential as a function of $R$ ).

In the spiral potential simulation, at $R=3 \mathrm{kpc}$ (top right panel in Figure 9), as gas flows into an arm, it first goes through a sharp density increase, followed by a slower decrease with respect to azimuth. The galaxy's rotation is to the right in this plot. The density profile is consistent with the shock scenario. At $R=4 \mathrm{kpc}$, the density contrast decreases (second right panel of Figure 9) and the profiles are fairly symmetric with respect to the peak value. This
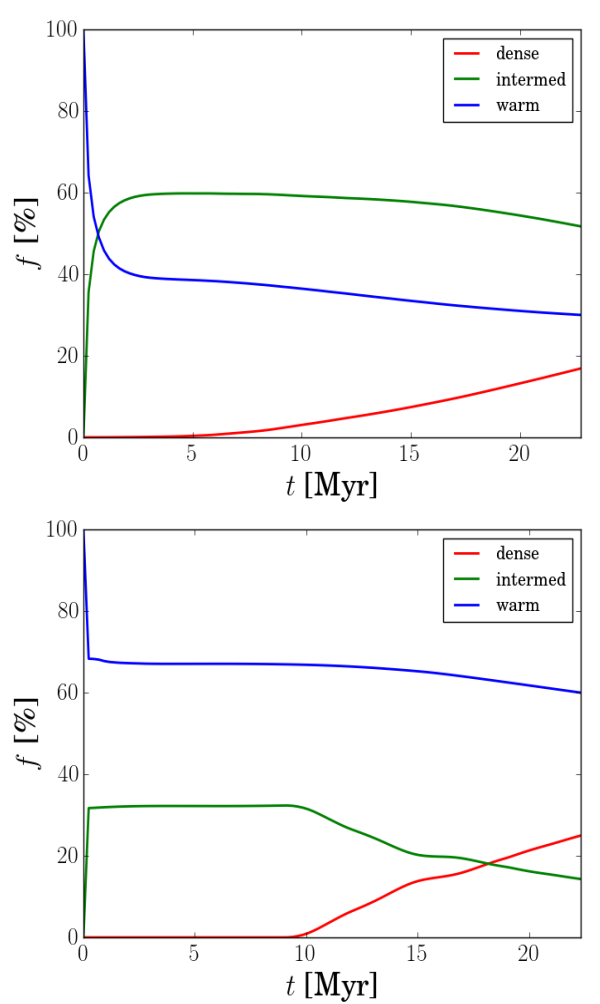

Figure 7. Evolution of the mass fraction of the cold, intermediate and warm gas phases for the $N$-body (top) and spiral potential simulations (bottom).

results from this region being closer to the corotation radius, where the gas flows into an arm from both sides.

At $R=5 \mathrm{kpc}$, the density profiles become less symmetric with respect to the maximum's location. On the peak's left-hand side, the density decreases more slowly with distance than on the rightand side. This behaviour is due to gas now entering from the arm's right side since at this radius, the arm rotates faster than the gas. At $R=7 \mathrm{kpc}$ (bottom right panel of Figure 9), the density profile has actually reversed with respect to the shape at $R=3 \mathrm{kpc}$. However, the density contrast is much smaller than in the inner regions. These profiles also show that the peak density values decrease with radius. The shape of the profiles are comparable to that expected in the spiral shock scenario (e.g. Fujimoto 1968; Roberts 1969).

From the azimuthal gas profiles of both models, it is worth noting that, even though different arm models were used, the azimuthal profiles in the $N$-body model at $R=4$ and $5 \mathrm{kpc}$ have qualitatively similar features to those at $R=3 \mathrm{kpc}$ in the spiral potential simulation.

\subsection{Gas Velocity Profiles and Streaming Motions}

The velocity profiles as a function of azimuth provide a more detailed picture of the gas dynamics in spiral arms. Such structures are expected to introduce significant perturbations to the gas velocities, so streaming motions should be important within them. Additionally, the gas velocities can be used to calculate the local divergence, which identifies regions of compression and expansion in the flows. The streaming motions and the local divergence are sensitive to the underlying nature of the flows. In this section, we analyse the velocity profiles as a function of azimuth at specific galactocentric radii and the amplitude of the streaming motions as a function of radius. 

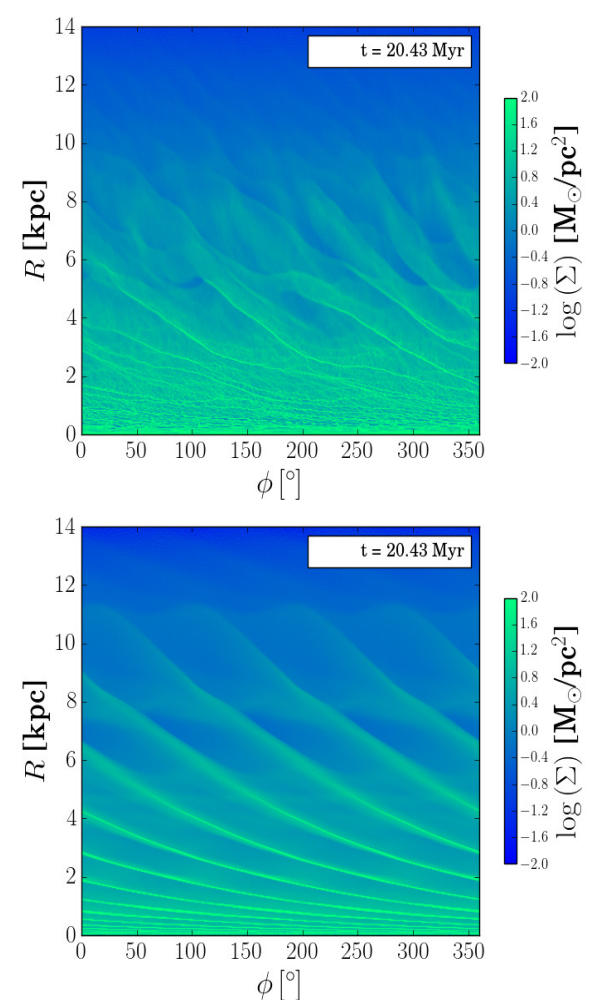

Figure 8. Gas surface density maps in a cylindrical projection for the $N$ body (top) and the spiral potential (bottom) simulations at $t=20.43 \mathrm{Myr}$. The galaxy rotates to the right hand side. The $N$-body simulation develops a richer structure compared to the spiral potential one at the selected time.

\subsubsection{Velocity profiles as a function of azimuth}

Figure 11 shows the velocity profiles of the $N$-body and the spiral potential simulations on the left and right columns, respectively, at $R=4,5$ and $7 \mathrm{kpc}$ on the top, middle, and bottom rows, respectively. The blue dots and red dots show, respectively, the radial $v_{R}$ and the relative azimuthal $v_{\phi(\text { rel })}$ components at the specified radius. We define this relative azimuthal velocity as $v_{\phi}$ (rel) $=\nu_{\phi}-\nu_{\phi}^{*}$. The solid curves show the background potential of the arms.

In the $N$-body simulation, the spiral arms are associated with sharp jumps in galactocentric velocity $v_{R}$ going from positive (outward) to negative (inward) values. At $R=4 \mathrm{kpc}$, the highest velocity is $v_{R} \approx 14 \mathrm{~km} \mathrm{~s}^{-1}$ and the lowest is almost $v_{R} \approx-14 \mathrm{~km} \mathrm{~s}^{-1}$. The profile at $R=5 \mathrm{kpc}$ has a similar range, but with more irregular profiles due to the more variable arm structure at this radius. At $R=7 \mathrm{kpc}$, the velocity profile's amplitude has decreased, but it is still sensitive to the arms. These results show that, in the $N$-body simulation, a sharp jump in $v_{R}$ correlates with the location of the spiral arms and the amplitude of the velocity perturbation decreases with radius. In the spiral potential simulation, at $R=4 \mathrm{kpc}$, the oscillation in $v_{R}$ has range within $\approx \pm 10 \mathrm{~km} \mathrm{~s}^{-1}$, which is similar to that of the smaller peaks in the $N$-body simulation at the same radius. The amplitude of the profile is lower at $R=5 \mathrm{kpc}$ due to its proximity to the corotation radius, but then shows a higher amplitude at $R=7 \mathrm{kpc}$. This is significantly different to the behaviour at the same radii in the $N$-body model.

In terms of the relative azimuthal component, the profile of the $N$-body simulation at $R=4 \mathrm{kpc}$ has values within $\pm 8 \mathrm{~km} \mathrm{~s}^{-1}$, although close to $\phi=250^{\circ}$ it reaches $\approx-10 \mathrm{~km} \mathrm{~s}^{-1}$. The $v_{\phi \text { (rel) }}$ pro- file's amplitude also decreases with galactocentric radius. In the spiral potential simulation, $v_{\phi}($ rel $)$ oscillates between $\approx \pm 7 \mathrm{~km} \mathrm{~s}^{-1}$ at $R=4 \mathrm{kpc}$. The amplitude is lowest near $R=5 \mathrm{kpc}$, but increases again at $R=7 \mathrm{kpc}$. At $R=7 \mathrm{kpc}, v_{\phi}$ (rel) peaks near the spiral potential minimum. A common feature in both simulations is that when $v_{R}$ reaches a maximum, $v_{\phi(\text { rel })}$ is at its lowest value, which can be expected from epicyclic-style motions (e.g. Baba et al. 2016).

In both models, the shape of the velocity profiles is qualitatively similar at positions inside the corotation radius. These profiles also show that gas on the downstream side, near an arm's potential minimum, tends to have significant inward motions $\left(v_{R}<0\right)$. The higher-density gas is usually located on this side, which shows that dense cloud-like structures forming downstream will have a negative $v_{R}$ component, but the densest gas will not necessarily have the most negative $v_{R}$

Figure 12 shows the azimuthal profiles of the divergence of the velocity field $(\nabla \cdot \vec{v})$ at $R=4 \mathrm{kpc}$ for the $N$-body and spiral potential simulations in the top and bottom panels, respectively. In both simulations, there is a tendency for $\nabla \cdot \vec{v}$ to vary from positive to negative values across a spiral arm. In both models, the arms drive a strong compression of the gas. However, in the $N$-body model, the divergence tends to be more negative downstream across an arm.

\subsubsection{Amplitude of the Streaming Motions}

The overall amplitude of the streaming motions can be measured by calculating the rms velocity $v_{\text {rms }}$ by averaging the velocities along the azimuthál direction. Figure 13 plots the rms velocity $v_{\mathrm{rms}}$ amplitude as a function of radius for the $N$-body model in the top panel and for the spiral potential in the bottom panel. It provides a global view of the average streaming velocities as a function of radius.

From a global perspective, Figure 13 shows some clear differences between the two simulations in the strength of the motions as a function of radius. In the $N$-body model, the amplitude of the streaming motions peaks near $R=4 \mathrm{kpc}$ and gradually decreases past this point. In the spiral potential model, the amplitude peaks near $R=2 \mathrm{kpc}$, then reaches a minimum slightly outside the corotation radius, and then reaches another maximum past this point. It is worth noting that the amplitude in $v_{R}$ in the spiral potential at positions outside the corotation radius increases to values comparable to those in the inner galaxy. This is interesting considering that the arms' amplitude decreases with radius. The velocities in the $\mathrm{N}$-body simulation tend to be lower than in the spiral potential one. A common feature in both models is that the amplitude in $v_{R}$ tends to be higher than in $v_{\phi}$.

\subsection{Gas Velocity Dispersion Profiles}

The gas velocity dispersion profiles can be used to trace regions where the flow has a more turbulent behaviour. As gas flows through a spiral arm, the flow can become turbulent after passing through shock regions (e.g. Bonnell et al. 2006). This shows that the velocity dispersion $\sigma_{v}$ is a function of azimuth, where the highest values are expected to be located in the arms.

Figure 14 shows the azimuthal profile of the gas velocity dispersion at $R=3 \mathrm{kpc}$ for the $N$-body and spiral potential simulations in the top and bottom panels respectively. The reference particles for this plot were selected in an annulus with radius of $3 \mathrm{kpc}$ and centred on the midplane. It has a radial width of $5.0 \mathrm{pc}$ and a height of $10 \mathrm{pc}$. The velocity dispersion is defined as $\sigma_{v}=\left(\bar{v}^{2}-\bar{v}^{2}\right)$, where $v$ is 

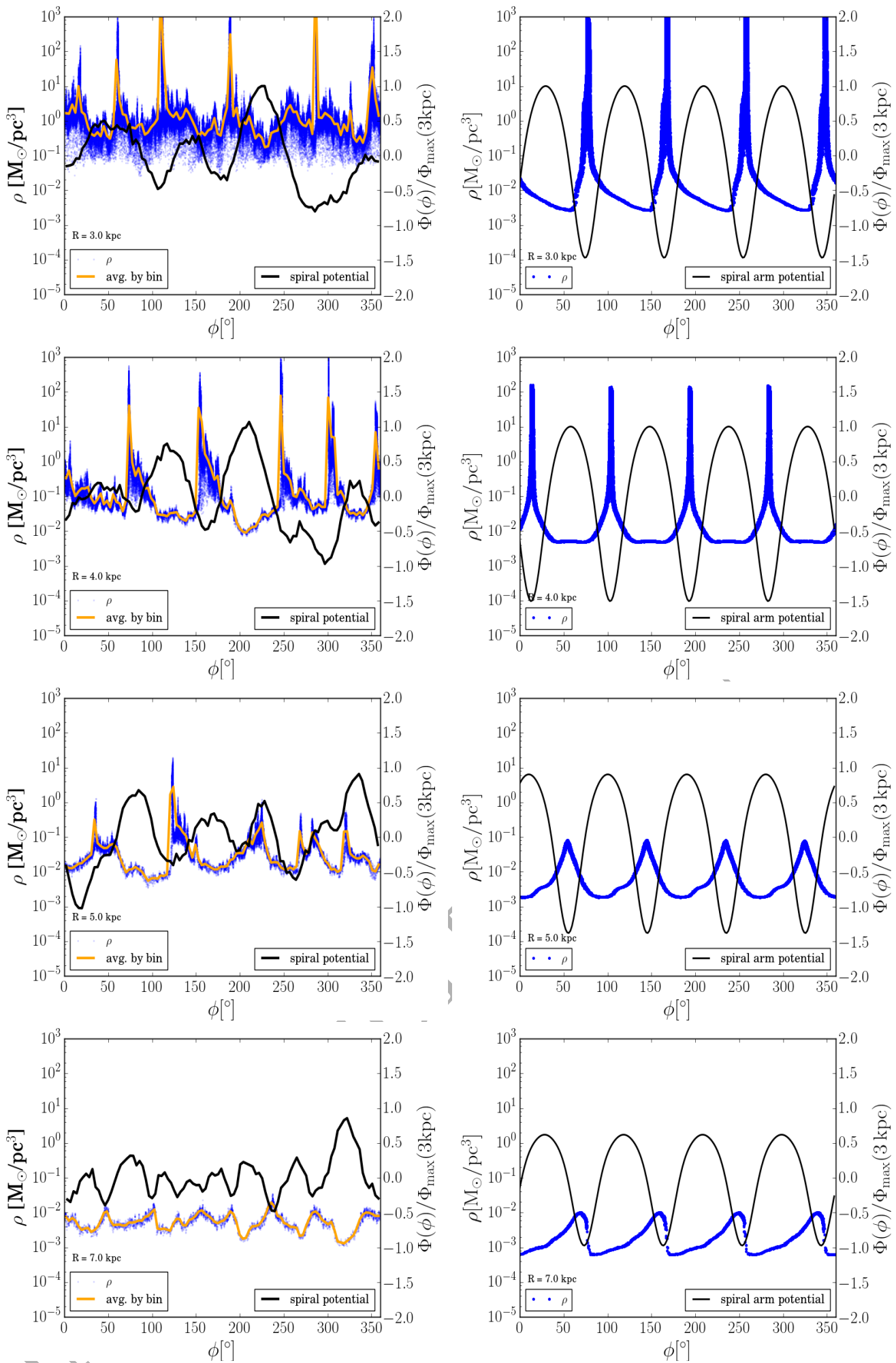

Figure 9. Azimuthal gas density profiles for the $N$-body (left column) and spiral potential (right column) simulations at $t=20.4$ Myr for $R=3 \mathrm{kpc}$ (top), $R=4 \mathrm{kpc}$ (second), $R=5 \mathrm{kpc}$ (third), $R=7 \mathrm{kpc}$ (bottom). The particles are selected in an annulus $50 \mathrm{pc}$ wide centred on the reference radius and within \pm 10 pc from the mid-plane. The orange curve in the plots at the left is the average density. In both columns, the black curve is the potential normalized to the peak at $R=3 \mathrm{kpc}$ to better visualize the variation with radius. 


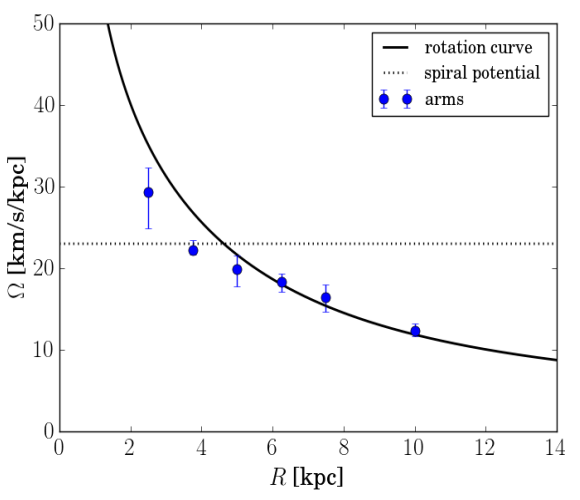

Figure 10. Angular frequencies of the circular velocity of the $N$-body model (black), the pattern speeds of its spiral arms (blue dots), and the pattern speed of the spiral potential (dotted). The $N$-body arm pattern speed is calculated by averaging the angular velocity of the stellar surface density peaks of the arms at the specified radius.

the magnitude of the relative velocity with respect to the reference particle; $\sigma_{v}$ is calculated using all the neighbours in the simulation within $100 \mathrm{pc}$ of each reference particle.

Both simulations show that $\sigma_{v}$ has peak values of a few $\mathrm{km} \mathrm{s}^{-1}$ in the spiral arms. The velocity dispersion tends to decrease with increasing galactocentric radius, as suggested by the profiles in the left panels of Figure 15 for the $N$-body and spiral potential simulations. In these plots, the velocity dispersion is calculated within a radius of $100 \mathrm{pc}$ from a given particle selected in a narrow angular region centred at $\phi=0^{\circ}$ and within $|z|<5 \mathrm{pc}$. The right panels of Figure 15 show the relative difference between the local gas surface density $\Sigma(R, 0)$ and the azimuthal average $\Sigma_{\text {mean }}(R)$. This difference is expressed as: $\Delta \Sigma=\Delta \Sigma(R, 0)-\Sigma_{\text {mean }}(R)$. This difference is calculated along a narrow angular region $2.7^{\circ}$ wide and using radial bins $3.5 \mathrm{pc}$ wide. The results of Figure 15 show a correspondence between regions with a high velocity dispersion and high surface density.

Our results show that the velocity dispersion tends to be enhanced in the spiral arms in both model galaxies. These results show that the spiral arms are effectively injecting turbulent motions regardless of the nature of the underlying spiral arms. However, we note that the magnitude of the dispersion may be underestimated due to the lack of energy injection by stellar and supernova feedback.

\section{DISCUSSION}

\subsection{Gas Disc Morphology and Cold Gas Distribution}

In the $N$-body simulation, the gas disc develops a clumpy and filamentary structure after the first few $\sim 10 \mathrm{Myr}$ of evolution. The gas traces well the stellar spiral arms, consistent with the $N$-body and hydrodynamics simulations of, for example, Clarke \& Gittins (2006); Wada et al,(2011); Mata-Chávez et al. (2014); Baba et al. (2017). An interesting result worth pointing out is that structure resembling "spurs" and "feathers" forms in the $N$-body simulation (Figure 5). These are confined to $R<3 \mathrm{kpc}$, where the local pattern speed does not corotate with the galaxy's circular velocity, so they could be driven by similar mechanisms as suggested by other works (e.g. Dobbs \& Bonnell 2006; Shetty \& Ostriker 2006). Although the medium becomes significantly fragmented due to self-gravity, these features are visible mainly on the downstream side of an arm.
In the spiral potential simulation, at the time shown in Figure 4 , the gas in the inter-arm regions has a very smooth surface density field. Other SPH simulations that have used the Cox \& Gómez (2002) potential such as Dobbs \& Bonnell (2006); Bonnell et al. (2013) and Smith et al. (2014) report the formation of "spurs" and "feathers". Other works that analysed hydrodynamical simulations in spiral potentials have reported these features (e.g. Kim \& Ostriker 2002, 2006; Shetty \& Ostriker 2006). The lack of structure in this simulation may be due to the following factors: first, we have assumed a slow rotation curve and a pattern speed that places the corotation radius well inside the disc, whereas other works have assumed a MW model with corotation at a much larger radius. Ramón-Fox (2019) performed a simulation with the potential of $\$ 2.3$ with cooling/heating but without including gas self-gravity. It shows that spur-like features appear after $\approx 40 \mathrm{Myr}$ and a rich variety of structures comparable to that of other simulations after 122 Myr. A second factor is that our method for initializing the spiral potential simulation allows the disc to evolve for an initial period of isothermal stabilisation, where the gas has no self-gravity. This produces a very smooth initial density field. Also the size scale for self-gravity is not necessarily that of the spiral arm. This simulation will likely form more structure after some time.

Both simulations show that cloud-like structures form in a "beads on a string" fashion. The $N$-body simulation shows, at least qualitatively, variations in the cloud separation along an arm whereas the spiral potential one shows a fairly regular separation. The simulations of Renaud et al. (2013) show that the separation tends to grow with time. The formation of clouds by gravitational collapse can explain a natural separation. However, Dobbs (2008) shows that the spacing is a result of agglomeration and orbit convergence as gas crosses a spiral shock, which is sensitive to the disc kinematics. This may explain some of the differences between our simulations. The more variable nature of the spiral arms and the gas kinematics the $N$-body model can translate into variations in the gas orbits that can explain the observed spacing. This is worth exploring in the future since observations suggest a fairly constant separation (e.g. Elmegreen \& Elmegreen 1983; Elmegreen et al. 2018).

In terms of the radial distribution of the ISM phases, the warm gas surface density has a fairly constant value as a function of radius, while the cold gas surface density falls exponentially with radius. These profiles and its range of surface density broadly agree with the observations of Bigiel \& Blitz (2012) and Druard et al. (2014). Our results show that these profiles' shape is not strongly sensitive to the galactic model, suggesting that the large-scale distribution of the ISM phases is not strongly dependent on the underlying nature of the galactic potential. A caveat of these profiles is that they average out the arm-to-arm variations; however, the azimuthal density profiles at several galactocentric radii in $\$ 3.3$ still show some qualitative similarities in both models. On the other hand, self-gravity plays a role in determining the peak density values in the arms. Stellar and supernova feedback also contribute to shaping the gas distribution. Although we cannot analyse their effect in our simulations, their absence may explain the thinness of the gas arms in our simulations.

\subsection{Large-Scale Gas Flows and Streaming Motions}

\subsubsection{Gas Flows across Spiral Arms}

Both Fujimoto (1968) and Roberts (1969) explored the behaviour of spiral arm shocks. Roberts (1969) found solutions for the density and velocity profiles in arm shocks, which predict that the shock is 

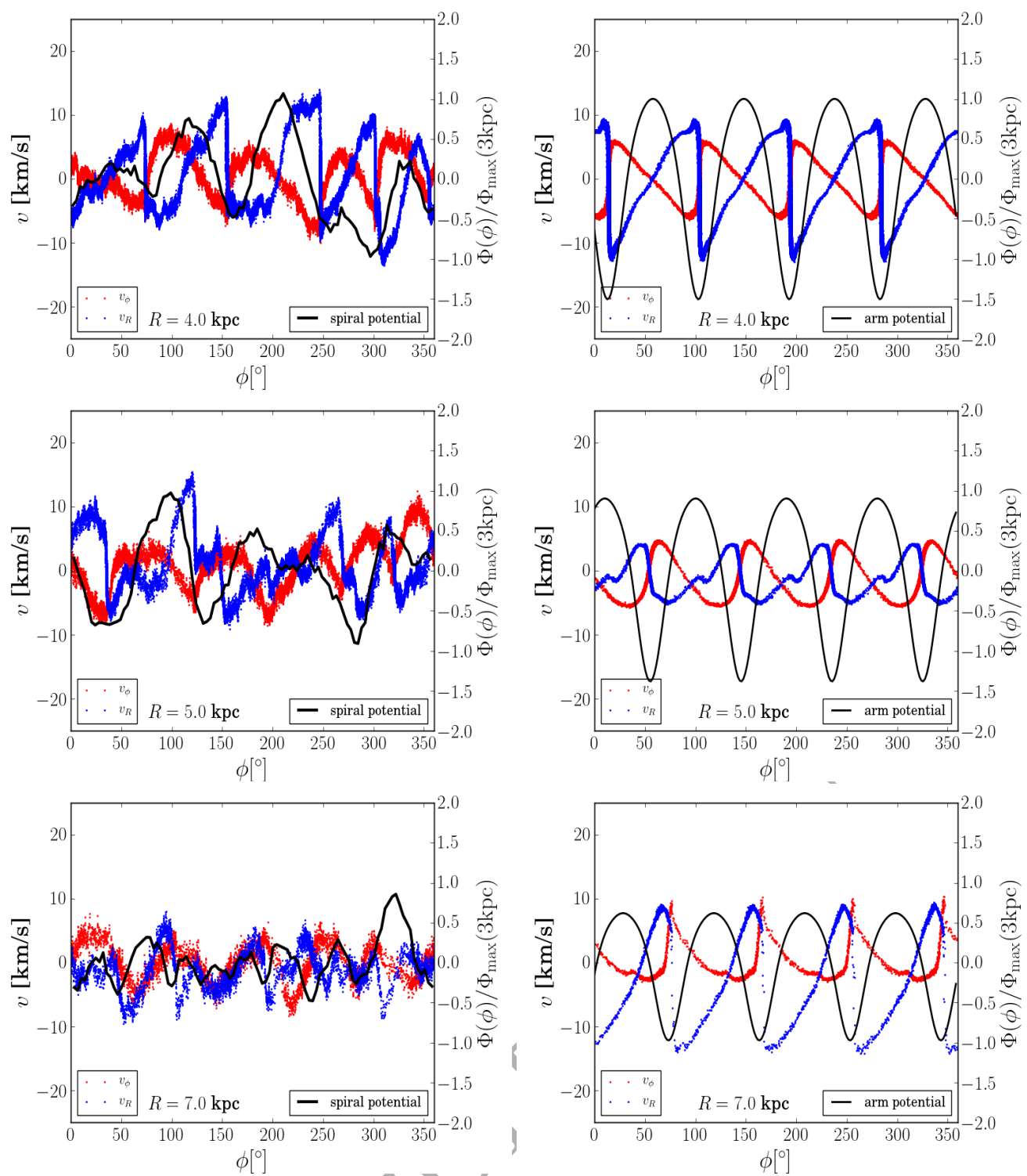

Figure 11. Azimuthal gas velocity profiles for the $N$-body, (left column) and spiral potential (right column) simulations. The blue and red dots show $v_{R}$ and $v_{\phi(\text { rel })}$, respectively. The black curves show the potential. The profiles are obtained at: $R=4 \mathrm{kpc}($ top), $R=5 \mathrm{kpc}($ middle), $R=7 \mathrm{kpc}(\mathrm{bottom})$ at $t=20.4$ Myr. The particles are chosen on an annulus $50 \mathrm{pc}$ wide centred in the reference radius and with $|z|<10 \mathrm{pc}$.

followed by a gradual decrease in density as a function of azimuth and that the velocity perpendicular to the arm first increases as it enters the arm and then falls sharply at the shock's location. The tangent velocity reaches a minimum near the shock. This model also predicts that as gas enters the arm, the shock forms before the gas reaches the potential minimum.

In the $N$-body simulation, the azimuthal density (left panels of Figure 9) and velocity, $v_{R}$ and $v_{\phi}$, profiles (left panels Figure 11) show some of these features, particularly at small galactocentric radii. Particularly, $v_{R}$ shows sharp jumps from positive to negative values at the same location as the density peaks. These features show small shifts with respect to the stellar arm's potential minimum, but without a specific trend. Our results broadly agree with those of Wada et al. (2011) and Baba et al. (2015). The velocity profiles in Baba et al. (2016) do not show the sharp jumps in $v_{R}$ that we find, which may be an effect of not including feedback in our simulations (see Appendix A).

In the spiral potential simulation, the density profile at $R=3$ kpc (Fig. 9) has some similarities to the Roberts (1969) model, but the density peak is closer to the arm's potential minimum and on the donwstream side, differing with the prediction of Roberts (1969). In the density profiles at larger radii, the density peaks are not significantly shifted from the potential minimum, likely due to its proximity to the corotation radius. This shift is also sensitive to the nature of the underlying arms (e.g Baba et al. 2015), the strength of the perturbation (e.g.Shetty \& Ostriker 2006), the number of arms (e.g. Gittins \& Clarke 2004; Kim \& Kim 2014), to whether the flow is steady-state or not (e.g. Dobbs 2007; Kovalenko \& Levi 1992), and to the phase of the infalling gas (e.g. Dobbs \& Bonnell 2007; Sormani et al. 2017). Dobbs (2007) finds that the shift decreases with increasing gas temperature and Dobbs \& Bonnell (2007) show 

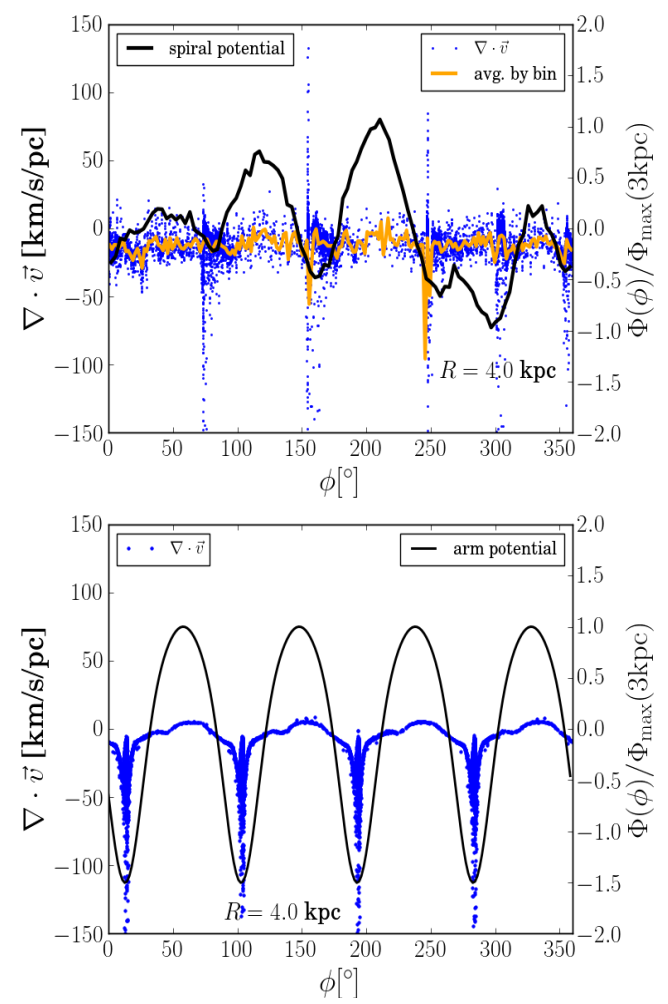

Figure 12. Divergence profile as a function of azimuth for the $N$-body (top) and spiral potential (bottom) simulations at $t=20.4$ Myr. Particles are selected from an annulus $10 \mathrm{pc}$ wide centred at $R=4.0 \mathrm{kpc}$ and with $|z|<5$ pc. $\nabla \cdot \vec{v}$ is calculated using the neighbours inside the smoothing kernel of a selected particle. In both models, the spiral arms strongly compress the gas.

that the warm and cold phases have different shifts from the minimum. It is likely that the smaller shifts in the density profiles in Figure 9 are a result of the gas entering an arm being warmer at the analysed time than in other similar simulations.

The $v_{R}$ and $v_{\phi}$ azimuthal profiles in the spiral potential simulation qualitatively agree with the Roberts (1969) model in the sense that the peak $v_{R}$ coincides with the $v_{\phi}$ minimum. Baba et al. (2016) explored the $v_{R}$ and $v_{\phi}$ profiles using an $m=2$ potential. Although we use an $m=4$ potential, we still find qualitatively similar profiles to theirs inside the corotation radius. However, we find some qualitative similarities in the shapes of the density and velocity profiles in several arms of our two simulations, which differs with the results of Baba et al. (2016). The velocity profiles outside the corotation radius of our potential simulation qualitatively agree with those of Kim \& Kim (2014) outside this radius.

Both of our simulations show that the spiral arms are effective in increasing the velocity dispersion, or turbulent flow, of the gas regardless of the underlying arm. In both models, $\sigma_{v}$ in the arms shows a tendency to decrease with galactocentric distance. The velocity dispersion results of our spiral potential model agrees with those of previous simulations with a similar model (e.g. Bonnell et al. 2006, 2013), although they did not perform full-galaxy simulations.

From a galactic-scale perspective, our simulations show that the $N$-body and spiral potential simulations have clear differences in the large-scale flow features: although both simulations agree in the sense that the maximum density in spiral arms decreases with galactocentric distance, they differ in terms of how the range of
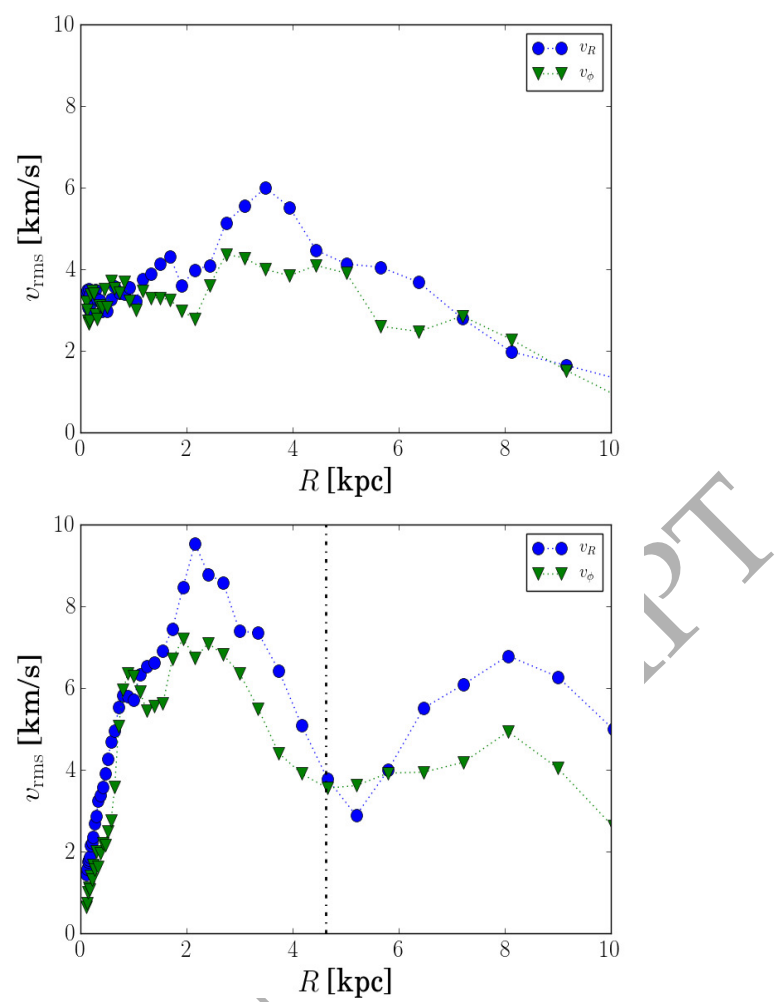

Figure 13. Gas radial and azimuthal $\mathrm{rms}$ velocities $v_{\mathrm{rms}}$ as a function of galactocentric radius for the $N$-body (top) and the spiral potential (bottom) simulations at $t=20.4$ Myr. The dashed-dotted line marks the corotation radius. $v_{\text {rms }}$ is calculated by averaging the velocity components azimuthally in annular bins and it measures the streaming motions' amplitude.

$v_{R}$ and $v_{\phi}$ values in the spiral arms vary with distance. In the $N$ body model, these values gradually decrease with distance, while in the spiral potential they reach a minimum near the corotation radius and increase past this point. This suggests that the large-scale features of the flow have some dependence on the nature of the galactic structure in agreement with previous works. However, on a smaller scale, the azimuthal flow profiles across individual arms show certain qualitative similarities, which has not been widely discussed in previous simulations. Our simulations show that spiral arms are effective in driving the formation of dense gas regions with high velocity dispersions. This appears to be not strongly dependent on the spiral arm model.

\subsubsection{Streaming Motions}

Both the $N$-body and spiral potential simulations show that the spiral arms drive significant non-circular gas motions. However, in the $N$-body simulation the amplitude of the streaming motions peaks in the inner galaxy and decreases at larger distances whereas in the spiral potential, the amplitude peaks in the inner galaxy, falls to a minimum near the corotation radius, and increases again at larger galactocentric radii. Both simulations show that the streaming velocities' amplitude in the radial direction tends to be higher than the azimuthal component.

Baba et al. (2016) also compared the gas kinematics in $N$-body and potential models of spiral arms in simulations of a Milky Waytype galaxy. Although we used a different galaxy model, we can still make a qualitative comparison. The simulations of Baba et al. 

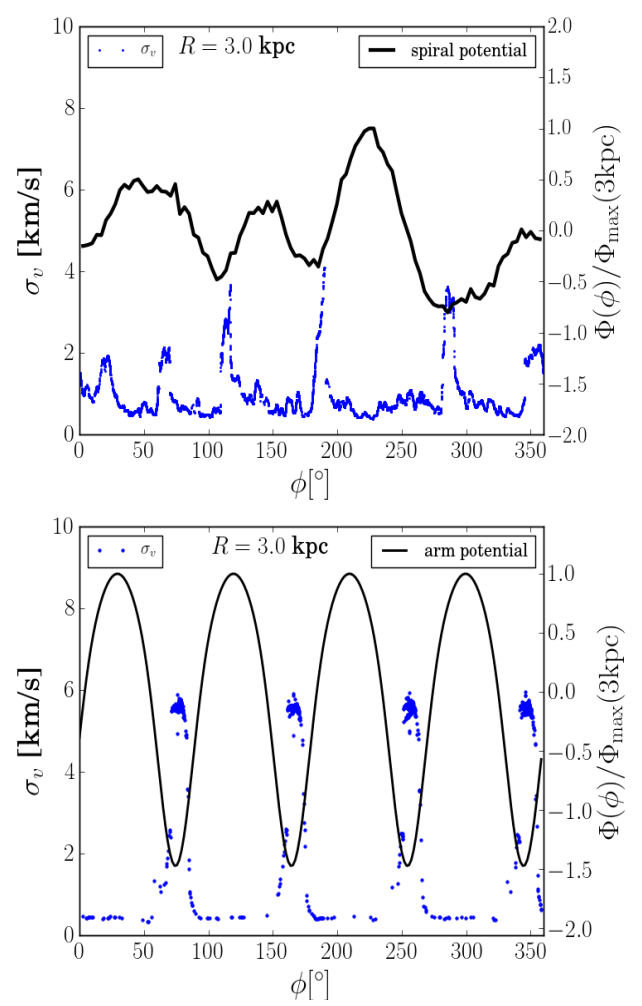

Figure 14. Velocity dispersion as a function of azimuth for the $N$-body (top) and the spiral potential (bottom) simulations at $t=20.4$ Myr. Particles are selected from an annulus at $R=3.0 \mathrm{kpc}, 5 \mathrm{pc}$ wide and with $|z|<5 \mathrm{pc}$; $\sigma_{v}$ is calculated using all the neighbours within $100 \mathrm{pc}$ from a reference particle. At this distance, $50^{\circ}$ corresponds to $2.62 \mathrm{kpc}$. In both models, the arms are enhancing the local velocity dispersion, which is indicative of their role in driving turbulent motions.

(2016) produce a larger amplitude of streaming motions than our simulations; their spiral potential simulation has a larger amplitude than their $N$-body model. In our simulations, we have ensured that the spiral potential amplitudes approximately match in the range $R=4-6 \mathrm{kpc}$, which results in a similar velocity peak-to-peak range in the two simulations. The velocity profiles of Baba et al. (2016) and Pettitt et al. (2020), which show some similarity to those in Figure 11, suggest that the streaming velocities in $y_{R}$ tend to be higher than in $v_{\phi}$. However, our results show that this is a global trend, and that there are differences between the two models in the behaviour of the amplitude as a function of radius.

Our $N$-body simulation also agrees with those of Wada et al. (2011) and Baba et al. (2009) in the sense that the streaming motions show arm-to-arm variations and are more unorganized when compared to a spiral potential simulation. Our spiral potential simulation qualitatively agrees with the velocity predictions of Gittins \& Clarke (2004), Kim \& Kim (2014), and Sormani et al. (2017).

The gas kinematics in M51, as reported by Meidt et al. (2013) using CO observations, show significant radial and azimuthal streaming motions: in the radial component, they gradually increase from $-20 \mathrm{~km} \mathrm{~s}^{-1}$ to $10 \mathrm{~km} \mathrm{~s}^{-1}$, with some oscillations in between; in the azimuthal component, the streaming motions vary between $\approx-10 \mathrm{~km} \mathrm{~s}^{-1}$ and $\approx 20 \mathrm{~km} \mathrm{~s}^{-1}$. The values in our simulations agree with these observations, but a direct comparison is not possible due to the fact that M51 shows evidence of interaction with a neighbouring galaxy. The $\mathrm{H}_{\mathrm{I}}$ kinematics of nearby spiral galaxies observed by Schmidt et al. (2016) show that $v_{R}$ oscillates around zero for several objects, ranging approximately between -20 $\mathrm{km} \mathrm{s}^{-1}$ and $20 \mathrm{~km} \mathrm{~s}^{-1}$, except for some particular cases. The gas in some galaxies show a net radial motion, which is attributed to a minor interaction, as predicted by some simulations (e.g. Ramón-Fox $\&$ Aceves 2020). The $v_{R}$ range of our simulations agrees with these values and it is not strongly sensitive to the spiral potential model.

Both the $N$-body and spiral potential simulations show that gas tends to move radially inward as it crosses an arm. Although the densest gas does not necessarily have the most negative $v_{R}$, the dense gas after the compression tends to have a negative $v_{R}$ component, at least inside the corotation radius. This agrees with the kinematics in the simulation of a Milky Way spiral arm by Ramón-Fox \& Bonnell (2018). This net inward motion has been observed in high-mass star forming regions in some arms of the Milky Way (e.g. Choi et al. 2014; Sato et al. 2014; Hachisuka et al. 2015). Although our results cannot be directly compared to these since we assumed a different galaxy model, it is worth noting that they show similar kinematic features. Another key result is that this inward motion is not strongly dependent on the underlying arm potential, it is more sensitive to the galactocentric distance in the $N$-body model and on the relative location with respect to the corotation radius in the spiral potential simulation. Our simulations also model the gas kinematics at distances outside the corotation radius, which may be relevant for characterizing spiral structure in extragalactic observations (e.g Clarke \& Gittins 2006; Kendall et al. 2011).

Our $N$-body simulation with cooling/heating was evolved for a short time (up to $\approx 40 \mathrm{Myr}$ ) and the arm's amplitude may not change significantly in this period. However, before this, the model was first evolved isothermally for $\approx 2.6$ orbital periods from an initially smooth exponential profile to allow spiral structure to form (see \$2.2). By visual inspection of its evolution, we see that the spiral arms evolve during this time. In this sense, our $N$-body model shows, at least qualitatively, features of dynamic arms (e.g. Wada et al.2011; Pettitt et al. 2015; Baba et al. 2015, 2016). We leave for future work to rerun our $N$-body simulation at a point where the spiral arm structure presents more significant changes or even dissipates (e.g. Baba et al. 2016; Pettitt et al. 2015), which can introduce different kinematic signatures in the local gas flows. The kinematics in dissipating arms certainly has implications for understanding spiral arms both in our Milky Way and other galaxies (e. g. Tchernyshyov et al. 2018).

\section{CONCLUSIONS}

We have performed high-resolution hydrodynamical simulations of a spiral galaxy with physical parameters representative of a lowmass galaxy like the Local Group spiral M33. Our simulations were aimed at comparing the large-scale flows and streaming motions driven by $N$-body and analytic potential models. Cooling and heating mechanisms were included to produce a two-phase ISM.

The results show that in both the $N$-body and spiral potential simulations, the spiral arms induce local shocks, an increase in kinetic energy and provide a means of compressing and expanding the gas. These are all crucial elements in forming molecular clouds and driving the necessary conditions for star formation. The simulations also show that the cold gas surface density falls with galactocentric distance, while the warm gas surface density has a fairly flat profile. This is qualitatively consistent with observations of nearby galaxies. The large-scale surface density profiles of the gas phases are 

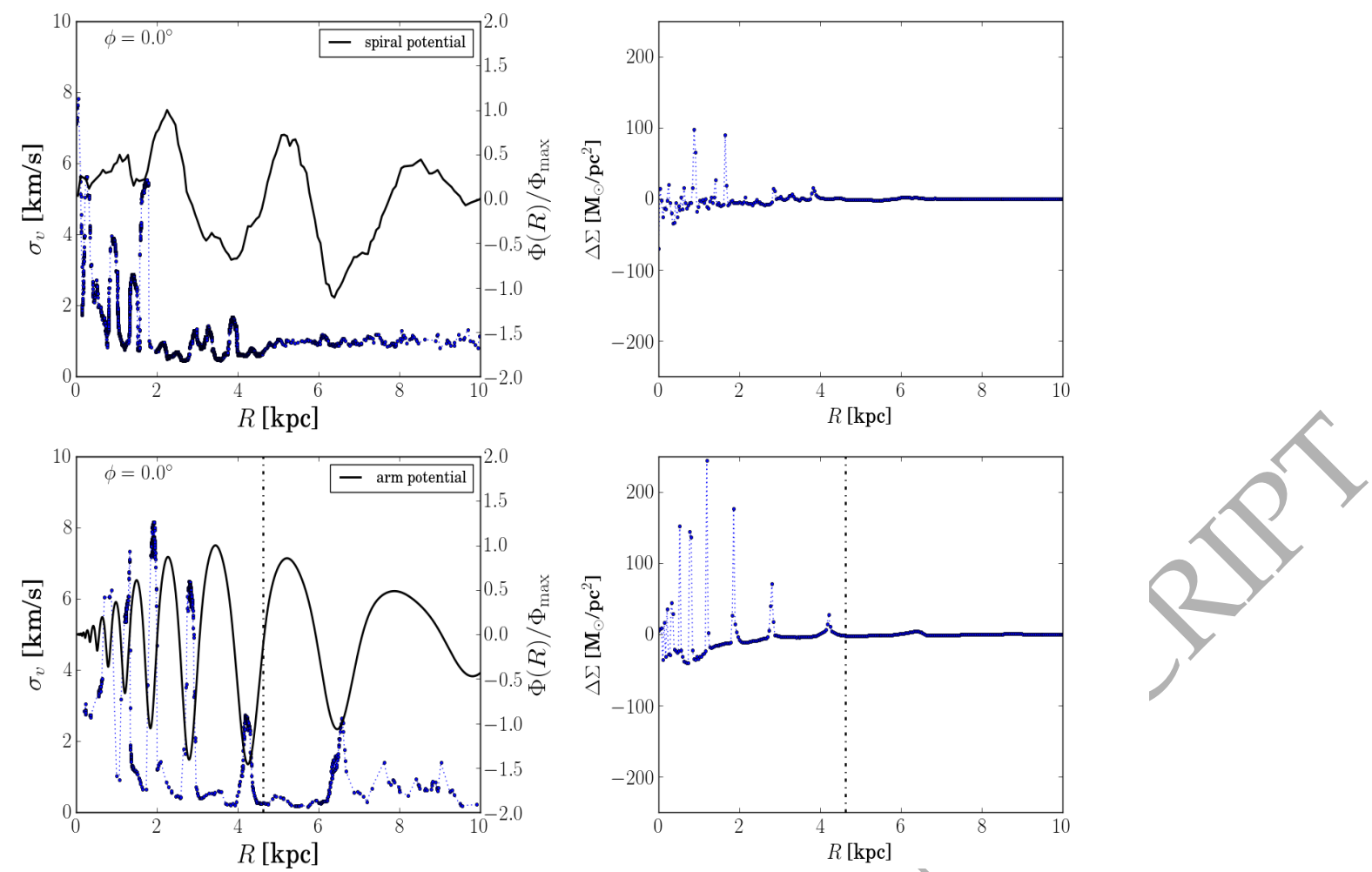

Figure 15. The left column shows the gas velocity dispersion $\sigma$ as a function of radius for the $N$-body (top left panel) and the spiral potential (bottom left panel) simulations at $t=20.4 \mathrm{Myr}$. The particles are selected from a narrow angular region centred at $\phi=0^{\circ}, \approx 0.57^{\circ}$ wide and with $|z|<5 \mathrm{pc}$. The velocity dispersion is calculated using neighbours within a radius of $100 \mathrm{pc}$ from a given particle. This length is larger than the typical smoothing length of the individual particles. The right column shows the difference between the local gas surface density and the azimuthal average as a function of radius, labelled as $\Delta \Sigma$. This shows the local density enhancements due to arms and clouds. There is a correspondence between the positions with high velocity dispersion and a higher gas surface density. The vertical dashed-dotted line marks the location of the corotation radius.

not strongly sensitive to the galaxy model. Both simulations show that clouds form on a string-like pattern along the spiral arm.

In terms of gas flows, the arms drive significant streaming velocities in both the radial and azimuthal directions. We find that cloud-like structures in the downstream side of an arm tends to have significant inward velocity components. The amplitude of the streaming velocities tends to be stronger in the radial component regardless of the model. The magnitude of these motions is consistent with values reported in observations of a sample of spiral galaxies. These motions have implications for observers mapping out the spiral structure of the Milky Way as well as for those modelling the kinematics of spiral galaxies. Their large-scale behaviour has some dependence on the galaxy model: in the $N$-body model, the streaming velocities peak in the inner galaxy and decrease with distance whereas in the spiral potential model, they decrease with distance up to the radius of corotation and increase with distance beyond this point.

\section{ACKNOWLEDGEMENTS}

We thank the anonymous referee for the insightful comments that improved this paper. FGR-F and IAB gratefully acknowledge support from the ERC ECOGAL project, grant agreement 291227, funded by the European Research Council under ERC2011-ADG. FGR-F also acknowledges a St. Leonards Scholarship from the University of St Andrews and support from the
Hyperstars project (funded by Région Paris Île-de-France DIMACAV+) at the final stages of this project. This research used NASA's Astrophysics Data System. This work used the DiRAC Complexity system, operated by the University of Leicester IT Services, which forms part of the STFC DiRAC HPC Facility (www.dirac.ac.uk). This equipment is funded by BIS National EInfrastructure capital grant ST/K000373/1 and STFC DiRAC Operations grant ST/K0003259/1. DiRAC is part of the National EInfrastructure. This work also used the HPC clusters at St Andrews. We warmly thank H. Fruchtl and I. Taylor for their technical support. We used SPLASH (Price 2007) for visualising SPH simulations. We are grateful to M. Petkova, W. Lucas, R. Smilgys and D. Forgan, and to the members of the Star Formation and Galaxies groups at St Andrews for their valuable input. FGR-F thanks members of the LMPA at the AIM for their valuable comments.

\section{DATA AVAILABILITY STATEMENT}

Part of the data underlying this article is available at the University of St Andrews Research Repository (http://hdl.handle.net/10023/17793). The data is also available on reasonable request to the corresponding author.

\section{REFERENCES}

Baba J., 2015, MNRAS, 454, 2954 
Baba J., Asaki Y., Makino J., Miyoshi M., Saitoh T. R., Wada K., 2009, ApJ, 706,471

Baba J., Morokuma-Matsui K., Egusa F., 2015, PASJ, 67, L4

Baba J., Morokuma-Matsui K., Miyamoto Y., Egusa F., Kuno N., 2016, MNRAS, 460, 2472

Baba J., Morokuma-Matsui K., Saitoh T. R., 2017, MNRAS, 464, 246

Bate M. R., Bonnell I. A., Price N. M., 1995, MNRAS, 277, 362

Benz W., Bowers R. L., Cameron A. G. W., Press W. H. ., 1990, ApJ, 348, 647

Bertin G., Lin C. C., 1996, Spiral structure in galaxies a density wave theory

Bigiel F., Blitz L., 2012, ApJ, 756, 183

Binney J., Tremaine S., 2008, Galactic Dynamics: Second Edition

Bonnell I. A., Dobbs C. L., Robitaille T. P., Pringle J. E., 2006, MNRAS, 365,37

Bonnell I. A., Dobbs C. L., Smith R. J., 2013, MNRAS, 430, 1790

Bournaud F., Elmegreen B. G., Teyssier R., Block D. L., Puerari I., 2010, MNRAS, 409, 1088

Burkert A., 2017, Mem. Soc. Astron. Italiana, 88, 533

Choi Y. K., Hachisuka K., Reid M. J., Xu Y., Brunthaler A., Menten K. M., Dame T. M., 2014, ApJ, 790, 99

Clarke C., Gittins D., 2006, MNRAS, 371, 530

Colombo D., et al., 2014, ApJ, 784, 4

Corbelli E., Salucci P., 2000, MNRAS, 311, 441

Cox D. P., Gómez G. C., 2002, ApJS, 142, 261

Cuddeford P., 1993, MNRAS, 262, 1076

Dehnen W., 1999, AJ, 118, 1201

Dobbs C., 2007, PhD thesis, University of St Andrews

Dobbs C. L., 2008, MNRAS, 391, 844

Dobbs C., Baba J., 2014, PASA, 31, e035

Dobbs C. L., Bonnell I. A., 2006, MNRAS, 367, 873

Dobbs C. L., Bonnell I. A., 2007, MNRAS, 376, 1747

Dobbs C. L., Theis C., Pringle J. E., Bate M. R., 2010, MNRAS, 403, 625

Dobbs C. L., Pettitt A. R., Corbelli E., Pringle J. E., 2018, MNRAS, 478, 3793

Druard C., et al., 2014, A\&A, 567, A118

Elmegreen B. G., 2002, ApJ, 577, 206

Elmegreen B. G., Elmegreen D. M., 1983, MNRAS, 203, 31

Elmegreen B. G., Elmegreen D. M., Efremov Y. N., 2018, ApJ, 863, 59

Forgan D. H., Ramón-Fox F. G., Bonnell I. A., 2018, MNRAS, 476, 2384

Freeman K. C., 1970, ApJ, 160, 811

Fujimoto M., 1968, in Non-stable Phenomena in Galaxies. p. 453

Gingold R. A., Monaghan J. J., 1977, MNRAS, 181, 375

Gittins D. M., Clarke C. J., 2004, MNRAS, 349, 909

Goldreich P., Lynden-Bell D., 1965, MNRAS, 130, 125

Gratier P., et al., 2010, A\&A, 522, A3

Hachisuka K., Choi Y. K., Reid M. J., Brunthaler A., Menten K. M., Sanna A., Dame T. M., 2015, ApJ, 800, 2

Hague P. R., Wilkinson M. I., 2015, ApJ, 800, 15

Hernquist L., 1990, ApJ, 356, 359

Julian W. H., Toomre A., 1966, ApJ, 146, 810

Kam Z. S., Carignan C., Chemin L., Amram P., Epinat B., 2015, MNRAS, 449, 4048

Kendall S., Kennicutt R. C., Clarke C., 2011, MNRAS, 414, 538

Kim Y., Kim W.-T., 2014, MNRAS, 440, 208

Kim W.-T., Ostriker E. C., 2002, ApJ, 570, 132

Kim W.-T., Ostriker E. C., 2006, ApJ, 646, 213

Koch E. W., et al., 2018, MNRAS, 479, 2505

Koch E. W., Rosolowsky E. W., Schruba A., Leroy A., Kepley A., Braine J., Dalcanton J., Johnson M. C., 2019, MNRAS, 485, 2324

Kovalenko I. G., Levi V. V., 1992, A\&A, 264, 406

Koyama H., Inutsuka S.-i., 2002, ApJ, 564, L97

Lin C. C., Shu F. H., 1964, ApJ, 140, 646

Lucas W. E., Bonnell I. A., Davies M. B., Rice W. K. M., 2013, MNRAS, 433,353

Mata-Chávez M. D., Gómez G. C., Puerari I., 2014, MNRAS, 444, 3756

McMillan P. J., Dehnen W., 2007, MNRAS, 378, 541

Meidt S. E., et al., 2013, ApJ, 779, 45

Meidt S. E., et al., 2018, ApJ, 854, 100
Monaghan J. J., 1992, ARA\&A, 30, 543

Monaghan J. J., Lattanzio J. C., 1985, A\&A, 149, 135

Navarro J. F., Frenk C. S., White S. D. M., 1997, ApJ, 490, 493

Pettitt A. R., Dobbs C. L., Acreman D. M., Bate M. R., 2015, MNRAS, 449, 3911

Pettitt A. R., Tasker E. J., Wadsley J. W., 2016, MNRAS, 458, 3990

Pettitt A. R., Dobbs C. L., Baba J., Colombo D., Duarte-Cabral A., Egusa F., Habe A., 2020, MNRAS, 498, 1159

Price D. J., 2007, Publ. Astron. Soc. Australia, 24, 159

Ramón-Fox F. G., 2019, PhD thesis, University of St Andrews

Ramón-Fox F. G., Aceves H., 2020, MNRAS, 491, 3908

Ramón-Fox F. G., Bonnell I. A., 2018, MNRAS, 474, 2028

Regan M. W., Vogel S. N., 1994, ApJ, 434, 536

Renaud F., et al., 2013, MNRAS, 436, 1836

Roberts W. W., 1969, ApJ, 158, 123

Sato M., et al., 2014, ApJ, 793, 72

Schmidt T. M., Bigiel F., Klessen R. S., de Blok W. J. G., 2016, MNRAS, 457,2642

Seigar M. S., 2011, ISRN Astronomy and Astrophysics, 2011, 725697

Sellwood J. A., Carlberg R. G., 1984, ApJ, 282, 61

Sellwood J. A., Shen J., Li Z., 2019, MNRAS, 486, 4710

Shetty R., Ostriker E. C., 2006, ApJ, 647, 997

Shu F. H., 2016, ARA\&A, 54, 667

Shu F. H., Milione V., Gebel W., Yuan C., Goldsmith D. W., Roberts W. W., 1972, ApJ, 173, 557

Smilgys R., Bonnell I. A., 2017, MNRAS, 472, 4982

Smith R. J., Glover S. C. O., Clark P. C., Klessen R. S., Springel V., 2014, MNRAS, 441, 1628

Sormani M. C., Sobacchi E., Shore S. N., Treß R. G., Klessen R. S., 2017, MNRAS, 471, 2932

Tchernyshyov K., Peek J. E. G., Zasowski G., 2018, AJ, 156, 248

Teuben P., 1995, in Shaw R. A., Payne H. E., Hayes J. J. E., eds, Astronomical Society of the Pacific Conference Series Vol. 77, Astronomical Data Analysis Software and Systems IV. p. 398

Vázquez-Semadeni E., Gómez G. C., Jappsen A. K., Ballesteros-Paredes J., González R. F., Klessen R. S., 2007, ApJ, 657, 870

Wada K., 2008, ApJ, 675, 188

Wada K., Baba J., Saitoh T. R., 2011, ApJ, 735, 1

Walter F., Brinks E., de Blok W. J. G., Bigiel F., Kennicutt Jr. R. C., Thornley M. D., Leroy A., 2008, AJ, 136, 2563

Wảng H.-H., Klessen R. S., Dullemond C. P., van den Bosch F. C., Fuchs B., 2010, MNRAS, 407, 705

van der Kruit P. C., Freeman K. C., 2011, ARA\&A, 49, 301

van der Kruit P. C., Searle L., 1981, A\&A, 95, 105

\section{APPENDIX A: EFFECT OF RESOLUTION ON THE AZIMUTHAL VELOCITY PROFILES}

We performed a low-resolution version of our simulations to analyse the effect of resolution on the $v_{R}$ and $v_{\phi}$ profiles as a function of azimuth. For the $N$-body model, we assigned $2 \times 10^{6}$ particles to the disc and 78420 particles to the bulge. For the gas, we used $2 \times 10^{6}$ particles. We assign the same number of gas particles to the spiral potential simulation. Each particle has a mass of $675 \mathrm{M}_{\odot}$.

Figure A1 shows the test results for the $N$-body model in the top panel and the spiral potential simulation in the bottom one. In the $N$ body model, the stellar potential profile has 4 arms, with amplitudes different to those of the higher resolution models. The velocity profiles for this simulation clearly show sharp jumps in $v_{R}$ near potential minima. The low-resolution spiral potential simulation shows an almost identical profile to that of Figure A1 at the same galactocentric radius, although the peak $v_{R}$ has slightly lower value at a lower resolution.

Our test shows that the low-resolution simulation still captures sharp jumps in $v_{R}$ and $v_{\phi}$ and produces similar velocity profiles 

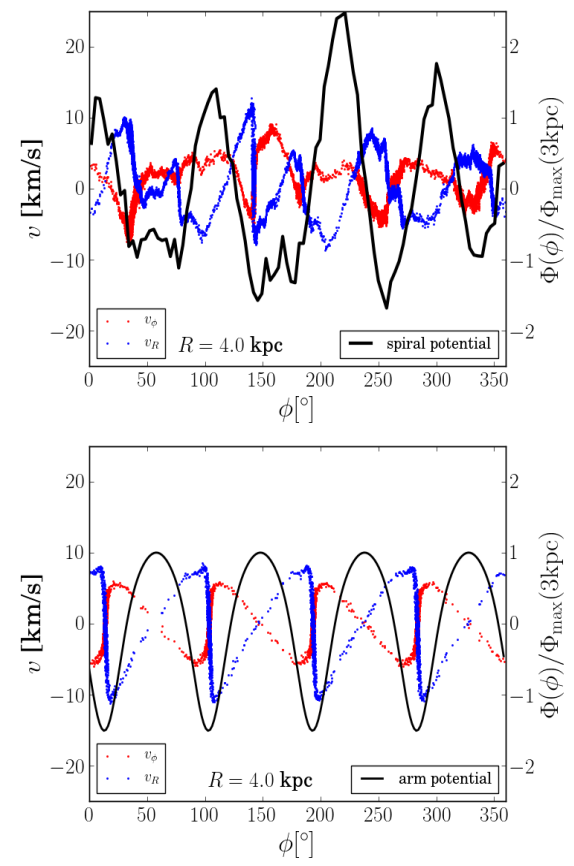

Figure A1. Azimuthal velocity profiles for the $N$-body (top) and the spiral potential (bottom) simulations at $R=4 \mathrm{kpc}$, at $t=20.4 \mathrm{Myr}$ with a resolution of $2 \times 10^{6}$ gas particles. Particles are selected with the same criteria as Figure 11.

as a function of azimuth. Other simulations (e.g. Baba et al. 2016) show less pronounced jumps. This difference may be attributed to feedback mechanisms, which are not included in our simulations. 